\title{
Strange and charm HVP contributions to the muon $(g-2)$ including QED corrections with twisted-mass
}

\section{fermions}

\section{Giusti, ${ }^{a, b}$ V. Lubicz, ${ }^{a, b}$ G. Martinelli, ${ }^{c}$ F. Sanfilippo ${ }^{b}$ and S. Simula ${ }^{b, 1}$ on behalf of ETM collaboration}

${ }^{a}$ Dipartimento di Matematica e Fisica, Università di Roma Tre,

Via della Vasca Navale 84, I-00146 Rome, Italy

${ }^{b}$ Istituto Nazionale di Fisica Nucleare, Sezione di Roma Tre,

Via della Vasca Navale 84, I-00146 Rome, Italy

${ }^{c}$ Dipartimento di Fisica, Università di Roma "La Sapienza" and INFN Sezione di Roma,

Piazzale Aldo Moro 5, 00185 Roma, Italy

E-mail: davide.giusti@uniroma3.it, vittorio.lubicz@uniroma3.it, guido.martinelli@roma1.infn.it, francesco.sanfilippo@roma3.infn.it, silvano.simula@roma3.infn.it

Abstract: We present a lattice calculation of the Hadronic Vacuum Polarization (HVP) contribution of the strange and charm quarks to the anomalous magnetic moment of the muon including leading-order electromagnetic corrections. We employ the gauge configurations generated by the European Twisted Mass Collaboration (ETMC) with $N_{f}=2+1+1$ dynamical quarks at three values of the lattice spacing ( $a \simeq 0.062,0.082,0.089 \mathrm{fm}$ ) with pion masses in the range $M_{\pi} \simeq 210-450 \mathrm{MeV}$. The strange and charm quark masses are tuned at their physical values. Neglecting disconnected diagrams and after the extrapolations to the physical pion mass and to the continuum limit we obtain: $a_{\mu}^{s}\left(\alpha_{\mathrm{em}}^{2}\right)=(53.1 \pm 2.5) \cdot 10^{-10}, a_{\mu}^{s}\left(\alpha_{\mathrm{em}}^{3}\right)=(-0.018 \pm 0.011) \cdot 10^{-10}$ and $a_{\mu}^{c}\left(\alpha_{\mathrm{em}}^{2}\right)=(14.75 \pm 0.56) \cdot 10^{-10}, a_{\mu}^{c}\left(\alpha_{\mathrm{em}}^{3}\right)=(-0.030 \pm 0.013) \cdot 10^{-10}$ for the strange and charm contributions, respectively.

KEYWORDS: Lattice field theory simulation, QCD Phenomenology

ARXIV EPRINT: 1707.03019

\footnotetext{
${ }^{1}$ Corresponding author.
} 


\section{Contents}

1 Introduction 1

2 Master formula 3

3 Lattice QCD simulations for $\boldsymbol{a}_{\mu}^{\text {had }} \quad 4$

3.1 Simulation details 4

3.2 Local versus conserved vector currents on the lattice 6

3.3 Perturbative QCD (pQCD) and the behavior of $V(t)$ at small $t \quad 8$

$\begin{array}{ll}3.4 \text { Ground-state identification } & 10\end{array}$

4 Strange and charm contributions: lowest order 13

5 Strange and charm contributions: e.m. corrections 18

6 Conclusions 23

A Non-perturbative estimate of the RCs $Z_{V}^{(\text {fact })}$ and $Z_{A}^{\text {(fact) }} \quad \mathbf{2 4}$

A.1 Axial Ward-Takahashi identity in the presence of electromagnetism 24

A.2 Determination of the RC $Z_{V} \quad 26$

$\begin{array}{lll}\text { A.3 Determination of the RCs } Z_{A} \text { and } Z_{P} / Z_{S} & 27\end{array}$

$\begin{array}{ll}\text { A.4 Numerical results } & 28\end{array}$

\section{Introduction}

The anomalous magnetic moment of the muon $a_{\mu} \equiv(g-2) / 2$ is known experimentally with an accuracy of the order of $0.54 \mathrm{ppm}$ [1], while the current precision of the Standard Model (SM) prediction is at the level of $0.4 \mathrm{ppm}[2]$. The tension of the experimental value with the SM prediction, $a_{\mu}^{\exp }-a_{\mu}^{\mathrm{SM}}=(28.8 \pm 8.0) \cdot 10^{-10}[2]$, corresponds to $\simeq 3.5$ standard deviations and might be an exciting indication of new physics.

The forthcoming $g-2$ experiments at Fermilab (E989) [3] and J-PARC (E34) [4] aim at reducing the experimental uncertainty by a factor of four, down to $0.14 \mathrm{ppm}$. Such a precision makes the comparison of the experimental value of $a_{\mu}$ with theoretical predictions one of the most important tests of the Standard Model in the quest for new physics effects.

It is clear that the experimental precision must be matched by a comparable theoretical accuracy. With a reduced experimental error, the uncertainty of the hadronic corrections will soon become the main limitation of this test of the SM. For this reason an intense research program is under way to improve the evaluation of the leading order hadronic contribution to $a_{\mu}$ due to the Hadronic Vacuum Polarization (HVP) correction to the oneloop diagram, $a_{\mu}^{\text {had }}\left(\alpha_{\mathrm{em}}^{2}\right)$, as well as to the next-to-leading-order hadronic ones. The latter include the $O\left(\alpha_{\mathrm{em}}^{3}\right)$ contribution of diagrams containing HVP insertions and the leading hadronic light-by-light (LBL) term [5]. 
The theoretical predictions for the hadronic contributions have been traditionally obtained from experimental data using dispersion relations for relating the HVP function to the experimental cross section data for $e^{+} e^{-}$annihilation into hadrons [6, 7]. An alternative approach was proposed in refs. [8-10], namely to compute $a_{\mu}^{\text {had }}\left(\alpha_{\mathrm{em}}^{2}\right)$ in Euclidean lattice QCD from the correlation function of two electromagnetic currents. In this respect an impressive progress in the lattice determinations of $a_{\mu}^{\text {had }}\left(\alpha_{\mathrm{em}}^{2}\right)$ has been achieved in the last few years [11-21] and very interesting attempts to compute also the LBL contribution are under way both on the lattice $[22,23]$ and via dispersion approaches and Chiral Perturbation Theory (ChPT) [24-26].

With the increasing precision of the lattice calculations, it becomes necessary to include electromagnetic (e.m.) and strong isospin breaking (IB) corrections (which contribute at order $O\left(\alpha_{\mathrm{em}}^{3}\right)$ and $O\left(\alpha_{\mathrm{em}}^{2}\left(m_{d}-m_{u}\right)\right)$, respectively) to the HVP. In this paper we present the results of a lattice calculation of the leading radiative e.m. corrections to the HVP contribution due to strange and charm quark intermediate states, obtained using the expansion method of refs. [27, 28]. Given the large statistical fluctuations, we are not in the position of giving results for the e.m. and IB corrections to the HVP contribution from the light up and down quarks, although we will give some details of our computation. For the same reason we do not have yet results for the disconnected contributions.

The main results of the present study for $a_{\mu}^{\text {had }}$ are for the lowest-order contributions

$$
\begin{aligned}
& a_{\mu}^{s} \equiv a_{\mu}^{s}\left(\alpha_{\mathrm{em}}^{2}\right)=(53.1 \pm 2.5) \cdot 10^{-10}, \\
& a_{\mu}^{c} \equiv a_{\mu}^{c}\left(\alpha_{\mathrm{em}}^{2}\right)=(14.75 \pm 0.56) \cdot 10^{-10}
\end{aligned}
$$

and for the e.m. corrections

$$
\begin{aligned}
& \delta a_{\mu}^{s} \equiv a_{\mu}^{s}\left(\alpha_{\mathrm{em}}^{3}\right)=(-0.018 \pm 0.011) \cdot 10^{-10} \\
& \delta a_{\mu}^{c} \equiv a_{\mu}^{c}\left(\alpha_{\mathrm{em}}^{3}\right)=(-0.030 \pm 0.013) \cdot 10^{-10}
\end{aligned}
$$

Our findings demonstrate that the expansion method of refs. [27, 28], which has been already applied successfully to the calculation of e.m. and strong IB corrections to meson masses [28, 29] and leptonic decays of pions and kaons [30, 31], works as well also in the case of the HVP contribution to $a_{\mu}$. This is reassuring about the feasibility of the determination of the leading e.m. and strong IB corrections to the HVP contribution from the up and down quarks, which is expected to be not negligible [5] and will be addressed in a separate work. For a recent calculation of these corrections, though at a large pion mass and at a fixed lattice spacing, see ref. [32], where, as expected, the strong IB effect is found to be at the percent level. In the strange and charm sectors the e.m. corrections (1.3)-(1.4) are found to be negligible with respect to present uncertainties.

The paper is organized as follows. In section 2 we introduce the basic quantities and notation. In section 3 we describe the lattice calculation and give the simulation details. In section 4 we present the calculation of the strange and charm contributions to the HPV at order $\mathcal{O}\left(\alpha_{\mathrm{em}}^{2}\right)$ and in section 5 the corresponding e.m. corrections at order $\mathcal{O}\left(\alpha_{\mathrm{em}}^{3}\right)$, which represent the original part of this work. Finally, section 6 contains our conclusions and outlooks for future developments. 


\section{Master formula}

The hadronic contribution $a_{\mu}^{\text {had }}$ to the muon anomalous magnetic moment at order $\alpha_{\mathrm{em}}^{2}$ can be related to the Euclidean space-time HVP function $\Pi\left(Q^{2}\right)$ by $[8-10]$

$$
a_{\mu}^{\mathrm{had}}=4 \alpha_{\mathrm{em}}^{2} \int_{0}^{\infty} d Q^{2} f\left(Q^{2}\right)\left[\Pi\left(Q^{2}\right)-\Pi(0)\right]
$$

where $Q$ is the Euclidean four-momentum and the kinematical kernel $f\left(Q^{2}\right)$ is given by

$$
f\left(Q^{2}\right)=\frac{1}{m_{\mu}^{2}} \frac{1}{\omega} \frac{1}{\sqrt{4+\omega^{2}}}\left(\frac{\sqrt{4+\omega^{2}}-\omega}{\sqrt{4+\omega^{2}}+\omega}\right)^{2}
$$

with $m_{\mu}$ being the muon mass and $\omega \equiv Q / m_{\mu}$.

The HVP form factor $\Pi\left(Q^{2}\right)$ is defined through the HVP tensor as

$$
\Pi_{\mu \nu}(Q) \equiv \int d^{4} x e^{i Q \cdot x}\left\langle J_{\mu}(x) J_{\nu}(0)\right\rangle=\left(\delta_{\mu \nu} Q^{2}-Q_{\mu} Q_{\nu}\right) \Pi\left(Q^{2}\right)
$$

where $\langle\ldots\rangle$ means the average of the $T$-product of the two electromagnetic (e.m.) currents over gluon and fermion fields and

$$
J_{\mu}(x) \equiv \sum_{f=u, d, s, c, \ldots} q_{f} \bar{\psi}_{f}(x) \gamma_{\mu} \psi_{f}(x)
$$

with $q_{f}$ being the electric charge of the quark with flavor $f$ in units of $e$.

In eq. (2.1) the subtracted HVP function $\Pi_{R}\left(Q^{2}\right) \equiv \Pi\left(Q^{2}\right)-\Pi(0)$ appears. This is due to the fact that the photon wave function renormalization constant absorbs the value of the photon self energy at $Q^{2}=0$ in order to guarantee that the e.m. coupling $\alpha_{\mathrm{em}}$ is the experimental one in the limit $Q^{2} \rightarrow 0$.

The HVP function $\Pi_{R}\left(Q^{2}\right)$ can be determined from the vector current-current Euclidean correlator $V(t)$ defined as

$$
V(t) \equiv \frac{1}{3} \sum_{i=1,2,3} \int d \vec{x}\left\langle J_{i}(\vec{x}, t) J_{i}(0)\right\rangle .
$$

Taking into account that $V(-t)=V(t)$ and choosing $Q$ along the time direction only, one has [33]

$$
\Pi_{R}\left(Q^{2}\right) \equiv \Pi\left(Q^{2}\right)-\Pi(0)=2 \int_{0}^{\infty} d t V(t)\left[\frac{\cos (Q t)-1}{Q^{2}}+\frac{1}{2} t^{2}\right] .
$$

Consequently the HVP contribution $a_{\mu}^{\text {had }}$ can be written as

$$
a_{\mu}^{\mathrm{had}}=4 \alpha_{\mathrm{em}}^{2} \int_{0}^{\infty} d t \tilde{f}(t) V(t),
$$

where $\tilde{f}(t)$ is given by [33]

$$
\tilde{f}(t) \equiv 2 \int_{0}^{\infty} d Q^{2} f\left(Q^{2}\right)\left[\frac{\cos (Q t)-1}{Q^{2}}+\frac{1}{2} t^{2}\right] .
$$


In what follows we will limit ourselves to the connected contributions to $a_{\mu}^{\text {had }}$. In this case each quark flavor $f$ contributes separately, i.e.

$$
a_{\mu}^{\mathrm{had}} \sim \sum_{f=u, d, s, c, \ldots}\left[a_{\mu}^{\mathrm{had}}(f)\right]_{(\mathrm{conn})} .
$$

For sake of simplicity we drop the label $f$ and the suffix (conn), but it is understood that hereafter we refer to the connected part of $a_{\mu}^{\text {had }}$ for a generic quark flavor $f$.

\section{Lattice QCD simulations for $a_{\mu}^{\text {had }}$}

The vector correlator $V(t)$ can be calculated on a lattice with volume $L^{3}$ and temporal extension $T$ at discretized values of $\bar{t} \equiv t / a$ from $-\bar{T} / 2$ to $\bar{T} / 2$ with $\bar{T}=T / a$. From now on all the "overlined" quantities are in lattice units.

A natural procedure is to split eq. (2.7) into two contributions, $a_{\mu}^{\text {had }}(<)$ and $a_{\mu}^{\text {had }}(>)$, corresponding to $0 \leq \bar{t} \leq \bar{T}_{\text {data }}$ and $\bar{t}>\bar{T}_{\text {data }}$, respectively. In the first contribution $a_{\mu}^{\text {had }}(<)$ the vector correlator is directly given by the lattice data, while for the second contribution $a_{\mu}^{\text {had }}(>)$ an analytic representation is required (see refs. $[17,18,20,21]$ ). If $\bar{T}_{\text {data }}$ is large enough that the ground-state contribution is dominant for $\bar{t}>\bar{T}_{\text {data }}$, one can write

$$
a_{\mu}^{\text {had }}=a_{\mu}^{\text {had }}(<)+a_{\mu}^{\text {had }}(>)
$$

with

$$
\begin{aligned}
& a_{\mu}^{\text {had }}(<)=4 \alpha_{\mathrm{em}}^{2} \sum_{\bar{t}=0}^{\bar{T}_{\text {data }}} \bar{f}(\bar{t}) \bar{V}(\bar{t}) \\
& a_{\mu}^{\text {had }}(>)=4 \alpha_{\mathrm{em}}^{2} \sum_{\bar{t}=\bar{T}_{\text {data }}+1}^{\infty} \bar{f}(\bar{t}) \frac{\bar{Z}_{V}}{2 \bar{M}_{V}} e^{-\bar{M}_{V} \bar{t}}
\end{aligned}
$$

where $\bar{Z}_{V} \equiv(1 / 3) \sum_{i=1,2,3}\left|\left\langle 0\left|J_{i}(0)\right| V\right\rangle\right|^{2}$ is the (squared) matrix element of the vector current operator (for the given quark flavor $f$ ) between the vector ground-state and the vacuum.

Note that $\bar{T}_{\text {data }}$ cannot be taken equal to $\bar{T} / 2$, because on the lattice the vector correlator possesses backward signals. In order to avoid them one has to choose an upper limit $\bar{T}_{\text {data }}$ sufficiently smaller than $\bar{T} / 2$. An important consistency check is that the sum of the two terms in the r.h.s. of eq. (3.1) should be almost independent of the specific choice of the value of $\bar{T}_{\text {data }}$, as it will be shown later in section 4 .

\subsection{Simulation details}

The gauge ensembles used in this work are the same adopted in ref. [34] to determine the up, down, strange and charm quark masses. We employed the Iwasaki action [35] for gluons and the Wilson Twisted Mass Action [36-38] for sea quarks. In order to avoid the mixing of strange and charm quarks in the valence sector we adopted a non-unitary set 


\begin{tabular}{|c|c|c|c|c|c|c|c|c|c|c|c|}
\hline ensemble & $\beta$ & $V / a^{4}$ & $a \mu_{\ell}$ & $a \mu_{\sigma}$ & $a \mu_{\delta}$ & $N_{c f g}$ & $a \mu_{s}$ & $a \mu_{c}$ & $M_{\pi}$ & $M_{K}$ & $M_{D}$ \\
\hline$A 30.32$ & \multirow[t]{8}{*}{1.90} & \multirow[t]{3}{*}{$32^{3} \times 64$} & 0.0030 & \multirow[t]{8}{*}{0.15} & \multirow[t]{8}{*}{0.19} & 150 & \multirow[t]{8}{*}{0.02363} & \multirow[t]{8}{*}{0.27903} & $275(10)$ & $568(22)$ & $2012(77)$ \\
\hline$A 40.32$ & & & 0.0040 & & & 100 & & & $316(12)$ & $578(22)$ & $2008(77)$ \\
\hline$A 50.32$ & & & 0.0050 & & & 150 & & & $350(13)$ & $586(22)$ & $2014(77)$ \\
\hline$A 40.24$ & & \multirow[t]{4}{*}{$24^{3} \times 48$} & 0.0040 & & & 150 & & & $322(13)$ & $582(23)$ & $2017(77)$ \\
\hline$A 60.24$ & & & 0.0060 & & & 150 & & & $386(15)$ & $599(23)$ & $2018(77)$ \\
\hline$A 80.24$ & & & 0.0080 & & & 150 & & & $442(17)$ & $618(24)$ & $2032(78)$ \\
\hline$A 100.24$ & & & 0.0100 & & & 150 & & & $495(19)$ & $639(24)$ & $2044(78)$ \\
\hline$A 40.20$ & & $20^{3} \times 48$ & 0.0040 & & & 150 & & & $330(13)$ & $586(23)$ & $2029(79)$ \\
\hline B25.32 & \multirow[t]{5}{*}{1.95} & \multirow[t]{4}{*}{$32^{3} \times 64$} & 0.0025 & \multirow[t]{5}{*}{0.135} & \multirow[t]{5}{*}{0.170} & 150 & \multirow[t]{5}{*}{0.02094} & \multirow[t]{5}{*}{0.24725} & $259 \quad(9)$ & $546(19)$ & $1942(67)$ \\
\hline B35.32 & & & 0.0035 & & & 150 & & & $302(10)$ & $555(19)$ & $1945(67)$ \\
\hline B55.32 & & & 0.0055 & & & 150 & & & 375 (13) & $578(20)$ & 1957 (68) \\
\hline B75.32 & & & 0.0075 & & & 80 & & & $436(15)$ & $599(21)$ & $1970(68)$ \\
\hline$B 85.24$ & & $24^{3} \times 48$ & 0.0085 & & & 150 & & & $468(16)$ & $613(21)$ & $1972(68)$ \\
\hline$D 15.48$ & \multirow[t]{3}{*}{2.10} & \multirow[t]{3}{*}{$48^{3} \times 96$} & 0.0015 & \multirow[t]{3}{*}{0.1200} & \multirow[t]{3}{*}{0.1385} & 100 & \multirow[t]{3}{*}{0.01612} & \multirow[t]{3}{*}{0.19037} & $223(6)$ & $529(14)$ & $1929(49)$ \\
\hline$D 20.48$ & & & 0.0020 & & & 100 & & & $256 \quad(7)$ & 535 (14) & $1933(50)$ \\
\hline D30.48 & & & 0.0030 & & & 100 & & & $312 \quad(8)$ & $550(14)$ & 1937 (49) \\
\hline
\end{tabular}

Table 1. Values of the simulated quark bare masses (in lattice units), of the pion, kaon and $D$ meson masses (in units of $\mathrm{MeV}$ ) for the 16 ETMC gauge ensembles with $N_{f}=2+1+1$ dynamical quarks used in this work (see ref. [34]). The values of the strange and charm quark bare masses $a \mu_{s}$ and $a \mu_{c}$, given for each gauge ensemble, correspond to the physical strange and charm quark masses determined in ref. [34]. The central values and errors of the pion, kaon and $D$-meson masses are evaluated using the bootstrap events of the eight branches of the analysis of ref. [34].

up [39] in which the valence strange and charm quarks are regularized as OsterwalderSeiler fermions [40], while the valence up and down quarks have the same action of the sea. Working at maximal twist such a setup guarantees an automatic $\mathcal{O}(a)$-improvement [38, 39].

We considered three values of the inverse bare lattice coupling $\beta$ and different lattice volumes, as shown in table 1 , where the number of configurations analyzed $\left(N_{c f g}\right)$ corresponds to a separation of 20 trajectories. At each lattice spacing, different values of the light sea quark masses have been considered. The light valence and sea quark masses are always taken to be degenerate. The bare masses of both the strange $\left(a \mu_{s}\right)$ and the charm $\left(a \mu_{c}\right)$ valence quarks are obtained, at each $\beta$, using the physical strange and charm masses and the mass renormalization constant (RC) determined in ref. [34]. The values of the lattice spacing are: $a=0.0885(36), 0.0815(30), 0.0619(18) \mathrm{fm}$ at $\beta=1.90,1.95$ and 2.10 , respectively.

In this work we made use of the bootstrap samplings elaborated for the input parameters of the quark mass analysis of ref. [34]. There, eight branches of the analysis were adopted differing in:

- the continuum extrapolation adopting for the scale parameter either the Sommer parameter $r_{0}$ or the mass of a fictitious PS meson made up of strange(charm)-like quarks;

- the chiral extrapolation performed with fitting functions chosen to be either a polynomial expansion or a Chiral Perturbation Theory (ChPT) Ansatz in the light-quark mass; 

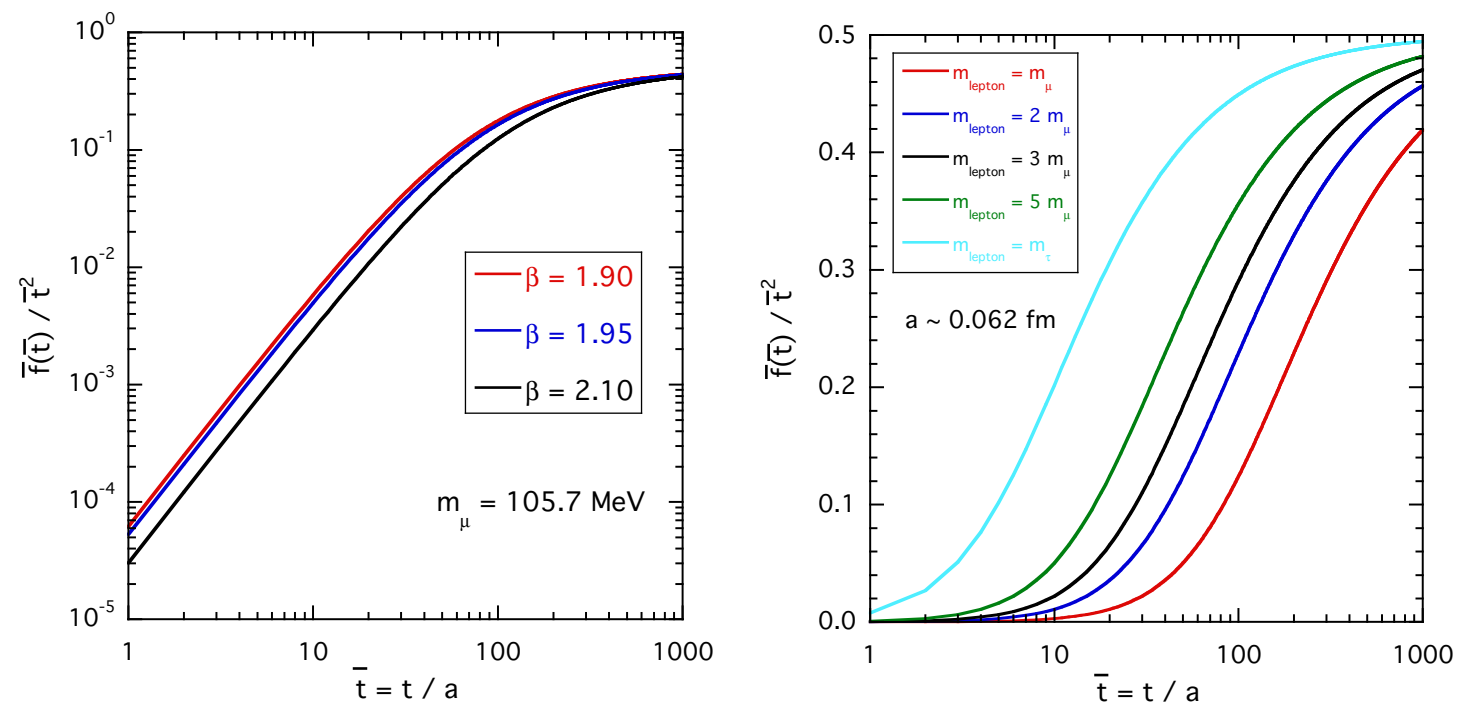

Figure 1. The kernel function $\bar{f}(\bar{t}) / \bar{t}^{2}$ versus the time distance $\bar{t}=t / a$ evaluated for the three values of the lattice spacing corresponding to the ETMC ensembles of table 1 (left panel) and for various values of the lepton mass ranging from the $\mu$ to the $\tau$ mass (right panel).

- the choice between the methods M1 and M2, which differ by $O\left(a^{2}\right)$ effects, used to determine in the RI'-MOM scheme the mass RC $Z_{m}=1 / Z_{P}$.

The kernel function $\bar{f}(\bar{t})$, appearing in eqs. (3.2)-(3.3), is explicitly given by

$$
\bar{f}(\bar{t})=\frac{4}{\bar{m}_{\mu}^{2}} \int_{0}^{\infty} d \omega \frac{1}{\sqrt{4+\omega^{2}}}\left(\frac{\sqrt{4+\omega^{2}}-\omega}{\sqrt{4+\omega^{2}}+\omega}\right)^{2}\left[\frac{\cos \left(\omega \bar{m}_{\mu} \bar{t}\right)-1}{\omega^{2}}+\frac{1}{2} \bar{m}_{\mu}^{2} \bar{t}^{2}\right]
$$

and can be easily calculated numerically at any value of $\bar{t}$ for given values of the muon mass in lattice units, $\bar{m}_{\mu} \equiv a m_{\mu}$. This is shown in figure 1 in the case of the muon at the three values of the lattice spacing of the ETMC ensembles of table 1 (left panel) and for various values of the lepton mass (right panel) ranging from the $\mu$ to the $\tau$ mass. The kernel function $\bar{f}(\bar{t})$ is proportional to $\bar{t}^{4}$ at small values of $\bar{t}$, diverges as $\bar{t}^{2}$ at large values of the time distance and has some sensitivity to the value of the lattice spacing. Instead it changes significantly with the mass of the lepton enhancing the role of the large-time behaviour of the vector correlator in the case of light leptons.

\subsection{Local versus conserved vector currents on the lattice}

The vector correlator $\bar{V}(\bar{t})$ can be calculated using either the lattice conserved vector current $J_{\mu}^{C}(x)$ or the local one $J_{\mu}(x)$. The latter needs to be renormalized and in our twistedmass setup the local vector current for each quark flavor $f$ is given by

$$
J_{\mu}(x)=q_{f} Z_{V} \bar{\psi}_{f}(x) \gamma_{\mu} \psi_{f}(x),
$$

where, being at maximal twist, the renormalization is multiplicative through the renormalization constant $Z_{V}$. 
The variation of the lattice action with respect to a vector rotation $\alpha_{V}(x)$ of the quark fields, i.e. $\psi(x) \rightarrow e^{i q_{f} \alpha_{V}(x)} \psi(x)$ and $\bar{\psi}(x) \rightarrow \bar{\psi}(x) e^{-i q_{f} \alpha_{V}(x)}$ (for any quark flavor $f$ ), provides the relevant Ward-Takahashi identity for the conserved current $J_{\mu}^{C}$ expressed in terms of the backward lattice derivative. In our twisted-mass setup one has

$$
\begin{aligned}
J_{\mu}^{C}(x)=q_{f} & \frac{1}{2}\left[\bar{\psi}_{f}(x)\left(\gamma_{\mu}-i \tau^{3} \gamma_{5}\right) U_{\mu}(x) \psi_{f}(x+a \hat{\mu})\right. \\
& \left.+\bar{\psi}_{f}(x+a \hat{\mu})\left(\gamma_{\mu}+i \tau^{3} \gamma_{5}\right) U_{\mu}^{\dagger}(x) \psi_{f}(x)\right] .
\end{aligned}
$$

According to the vector Ward-Takahashi identity the polarization tensor $\left\langle J_{\mu}^{C}(x) J_{\nu}^{C}(y)\right\rangle$ is not transverse because of the contact term arising from the vector rotation of the conserved current $J_{\nu}^{C}(y)$, which generates the backward lattice derivative of the tadpole operator and is power divergent as $1 / a^{3}$. Thus, in the case of two conserved currents the transverse HVP tensor is defined as

$$
\Pi_{\mu \nu}^{C C}(x, y) \equiv\left\langle J_{\mu}^{C}(x) J_{\nu}^{C}(y)\right\rangle-\frac{1}{a^{3}} \delta_{\mu \nu} \delta_{x y}\left\langle T_{\nu}(y)\right\rangle,
$$

where the tadpole operator is explicitly given by

$$
\begin{aligned}
T_{\nu}(y)=q_{f}^{2} & \frac{1}{2}\left[\bar{\psi}_{f}(y)\left(\gamma_{\nu}-i \tau^{3} \gamma_{5}\right) U_{\nu}(y) \psi_{f}(y+a \hat{\nu})\right. \\
& \left.-\bar{\psi}_{f}(y+a \hat{\nu})\left(\gamma_{\nu}+i \tau^{3} \gamma_{5}\right) U_{\nu}^{\dagger}(y) \psi_{f}(y)\right] .
\end{aligned}
$$

On the contrary, in the case of one conserved and one local currents there is no contact term because the vector rotation of the local current (3.5) is zero. One gets

$$
\Pi_{\mu \nu}^{C L}(x, y) \equiv\left\langle J_{\mu}^{C}(x) J_{\nu}(y)\right\rangle,
$$

which is transverse only with respect to the $\mu$ index (i.e., $\partial_{\mu}^{b} \Pi_{\mu \nu}^{C L}(x, y)=0$, where $\partial_{\mu}^{b}$ is the backward lattice derivative).

In the case of two local currents the polarization tensor $\left\langle J_{\mu}(x) J_{\nu}(y)\right\rangle$ is not transverse. The mixing pattern of the product of two local currents with all possible operators with equal and lower dimensions has been investigated for the twisted-mass setup in ref. [41]. The outcome is that at maximal twist one has

$$
\begin{aligned}
\int d^{4} x e^{i Q x}\left\langle J_{\mu}(x) J_{\nu}(0)\right\rangle= & \Pi_{\mu \nu}(Q)+\delta_{\mu \nu} \mathcal{Z}_{1}\left(\frac{1}{a^{2}}-S_{6}+\frac{S_{5}^{2}}{2}\right)+\delta_{\mu \nu} \mathcal{Z}_{m} m^{2} \\
& +\delta_{\mu \nu} \mathcal{Z}_{L} Q^{2}+\left(\delta_{\mu \nu} Q^{2}-Q_{\mu} Q_{\nu}\right) \mathcal{Z}_{T}+\mathcal{O}\left(a^{2}\right),
\end{aligned}
$$

where $\Pi_{\mu \nu}(Q)$ is the transverse polarization tensor, $S_{5}$ and $S_{6}$ are the vacuum expectation values of the dimension- 5 and -6 terms of the Symanzik expansion of the twisted-mass action, $m$ is the (twisted) quark mass and the quantities $\mathcal{Z}_{1}, \mathcal{Z}_{m}, \mathcal{Z}_{L}$ and $\mathcal{Z}_{T}$ are mixing coefficients.

In the r.h.s. of eq. (3.10) the second and third terms do not depend on $Q$, while the fourth and fifth terms are $Q$-dependent. The former ones can be eliminated by considering the subtracted form

$$
\begin{aligned}
\int d^{4} x\left(e^{i Q x}-1\right)\left\langle J_{\mu}(x) J_{\nu}(0)\right\rangle= & \Pi_{\mu \nu}(Q)+\delta_{\mu \nu} \mathcal{Z}_{L} Q^{2} \\
& +\left(\delta_{\mu \nu} Q^{2}-Q_{\mu} Q_{\nu}\right) \mathcal{Z}_{T}+\mathcal{O}\left(a^{2}\right)
\end{aligned}
$$


where we have considered that $\Pi_{\mu \nu}(0)=0$ in the infinite volume limit [33]. Choosing $Q$ along the time direction only with $\mu=\nu=i=1,2,3$ one has

$$
\int d t\left(e^{i Q t}-1\right) \int d^{3} x\left\langle J_{i}(x) J_{i}(0)\right\rangle=\Pi_{i i}(Q)+\left(\mathcal{Z}_{L}+\mathcal{Z}_{T}\right) Q^{2}+\mathcal{O}\left(a^{2}\right) .
$$

Using eqs. (2.3) and (2.5) and taking into account that $V(t)=V(-t)$, one obtains

$$
2 \int_{0}^{\infty} d t \frac{\cos (Q t)-1}{Q^{2}} V(t)=\Pi\left(Q^{2}\right)+\mathcal{Z}_{L}+\mathcal{Z}_{T}+\mathcal{O}\left(a^{2}\right) .
$$

In the renormalized HVP function $\left[\Pi\left(Q^{2}\right)-\Pi(0)\right]$ the term $\left(\mathcal{Z}_{L}+\mathcal{Z}_{T}\right)$ cancels out, so that eq. (2.6) is recovered and the $\mathcal{O}(a)$-improvement of the renormalized HVP function is guaranteed.

In this work the local version of the vector current is adopted (see later eq. (3.23) in section 3.4).

\subsection{Perturbative QCD (pQCD) and the behavior of $V(t)$ at small $t$}

The HVP function $\Pi_{R}\left(Q^{2}\right)$ obeys the (once subtracted) dispersion relation

$$
\Pi_{R}\left(Q^{2}\right) \equiv \Pi\left(Q^{2}\right)-\Pi(0)=\frac{1}{12 \pi^{2}} \int_{s_{\mathrm{thr}}}^{\infty} d s \frac{Q^{2}}{s\left(s+Q^{2}\right)} R^{\mathrm{had}}(s),
$$

where $R^{\text {had }}(s)$ is related to the (one photon) $e^{+} e^{-}$annihilation cross section into hadrons, $\sigma^{\text {had }}(s)$, by

$$
\sigma^{\text {had }}(s)=\frac{4 \pi \alpha_{\mathrm{em}}^{2}}{s} R^{\text {had }}(s)
$$

with $s$ being the center-of-mass energy and $s_{\mathrm{thr}}=4 M_{\pi}^{2}$.

The pQCD prediction for $R^{\text {had }}(s)$ is known up to three loops including mass corrections [42]. Here we limit ourselves to the lowest order prediction, which, for each quark flavor $f$, reads as

$$
R^{\mathrm{pQCD}}(s)=q_{f}^{2} N_{c} \sqrt{1-\frac{4 m^{2}}{s}}\left(1+\frac{2 m^{2}}{s}\right) \theta\left(s-4 m^{2}\right)+\mathcal{O}\left(\alpha_{s}\right),
$$

where $m$ is the on-shell quark mass. Inserting eq. (3.16) into eq. (3.14) one obtains

$$
\Pi_{R}^{\mathrm{pQCD}}\left(Q^{2}\right)=\frac{q_{f}^{2} N_{c}}{12 \pi^{2}}\left[x^{2}-\frac{5}{3}+\left(2-x^{2}\right) \sqrt{1+x^{2}} \ln \left(\frac{1+\sqrt{1+x^{2}}}{x}\right)\right],
$$

where $x \equiv 2 m / Q$. The behavior of $\Pi_{R}^{\mathrm{pQCD}}\left(Q^{2}\right)$ at large $Q^{2}$ is given by

$$
\begin{aligned}
\Pi_{R}^{\mathrm{pQCD}}\left(Q^{2}\right) \underset{Q^{2} \rightarrow \infty}{\longrightarrow} \frac{q_{f}^{2} N_{c}}{12 \pi^{2}}\{ & \ln \left(\frac{Q^{2}}{m^{2}}\right)-\frac{5}{3}+6 \frac{m^{2}}{Q^{2}} \\
& \left.-3 \frac{m^{4}}{Q^{4}}\left[1+2 \ln \left(\frac{Q^{2}}{m^{2}}\right)\right]+\mathcal{O}\left(\frac{m^{6}}{Q^{6}}\right)\right\},
\end{aligned}
$$

which exhibits a logarithmic divergence. 
In the continuum the vector correlator (2.5) can be obtained simply by taking the Fourier transform of the spatial components of the HVP tensor (2.3). Choosing $Q$ along the time direction only, one gets

$$
V(t) \equiv \int_{-\infty}^{\infty} d Q e^{-i Q t} \frac{1}{3} \sum_{i=1,2,3} \Pi_{i i}(Q) \underset{t>0}{\longrightarrow} \int_{-\infty}^{\infty} d Q e^{-i Q t} Q^{2} \Pi_{R}\left(Q^{2}\right) .
$$

Using eq. (3.14) one has

$$
\begin{aligned}
V(t) & \underset{t>0}{\longrightarrow} \frac{1}{12 \pi^{2}} \int_{s_{\mathrm{thr}}}^{\infty} d s \frac{1}{s} R^{\mathrm{had}}(s) \int_{-\infty}^{\infty} d Q e^{-i Q t} \frac{Q^{4}}{s+Q^{2}} \\
& \longrightarrow \frac{1}{24 \pi^{2}} \int_{s_{\mathrm{thr}}}^{\infty} d s \sqrt{s} R^{\mathrm{had}}(s) e^{-\sqrt{s} t} .
\end{aligned}
$$

Consequently, using the pQCD result (3.16) for $R^{\text {had }}(s)$ (including the quark mass threshold $s_{\text {thr }}=4 m^{2}$ ) the pQCD prediction for $V(t)$ is given by

$$
\begin{aligned}
V^{\mathrm{pQCD}}(t) \underset{t>0}{\longrightarrow} & \frac{2 q_{f}^{2} N_{c}}{3 \pi^{2}} m^{3} \int_{1}^{\infty} d y y^{2} \sqrt{1-\frac{1}{y^{2}}}\left(1+\frac{1}{2 y^{2}}\right) e^{-2 m t y} \\
= & \frac{q_{f}^{2} N_{c}}{6 \pi^{2}}\left\{\frac{1}{t^{3}} e^{-2 m t}\left(1+2 m t+2 m^{2} t^{2}\right)\right. \\
& \left.+4 m^{3} \int_{1}^{\infty} d y y^{2}\left[\sqrt{1-\frac{1}{y^{2}}}\left(1+\frac{1}{2 y^{2}}\right)-1\right] e^{-2 m t y}\right\} .
\end{aligned}
$$

Note that $e^{-2 m t}\left(1+2 m t+2 m^{2} t^{2}\right)=1+\mathcal{O}\left(m^{3} t^{3}\right)$ and therefore at small values of $t$ the vector correlator $V^{\mathrm{pQCD}}(t)$ is dominated by a mass-independent term, namely

$$
V^{\mathrm{pQCD}}(t) \underset{t \ll 1 / m}{\longrightarrow} \frac{q_{f}^{2}}{2 \pi^{2}} \frac{1}{t^{3}}+\mathcal{O}\left(m^{3}, m^{4} t\right)
$$

which represents also the vector correlator $V^{\mathrm{pQCD}}(t)$ in the massless limit.

In figure 2 we compare the pQCD predictions (3.21) and (3.22) with the vector correlator $V(t)$ obtained using the ETMC ensembles A30.32, B25.32 and D20.48, which share an approximate common value of the light-quark mass $m_{\ell} \simeq 12 \mathrm{MeV}$ and differ only in the values of the lattice spacing. It can be clearly seen that at small values of $t$ the lattice data match nicely the (lowest order) pQCD prediction. The inclusion of the radiative corrections from ref. [42] leads to an effect of the order of $\approx 10 \%$, which does not modify the quality of the agreement shown in figure 2 .

A closer look to figure 2 shows that the matching with pQCD is present up to time distances of $\approx 1 \mathrm{fm}$ (the agreement can be extended in the case of the strange vector correlator by including the corrections due to the strange quark mass), which corresponds to $1 / \Lambda_{\mathrm{QCD}}$ with $\Lambda_{\mathrm{QCD}} \approx 300 \mathrm{MeV}$, i.e., the agreement is observed down to energy scales of the order of $\Lambda_{\mathrm{QCD}}$. One would expect that pQCD works at $t \ll 1 / \Lambda_{\mathrm{QCD}}$ or $Q \gg \Lambda_{\mathrm{QCD}}$. The fact that instead the matching appears to work at larger time distances is a nice manifestation of the quark-hadron duality à $l a S V Z$, which states that the sum of the contributions of the excited states is dual to the pQCD behaviour. 

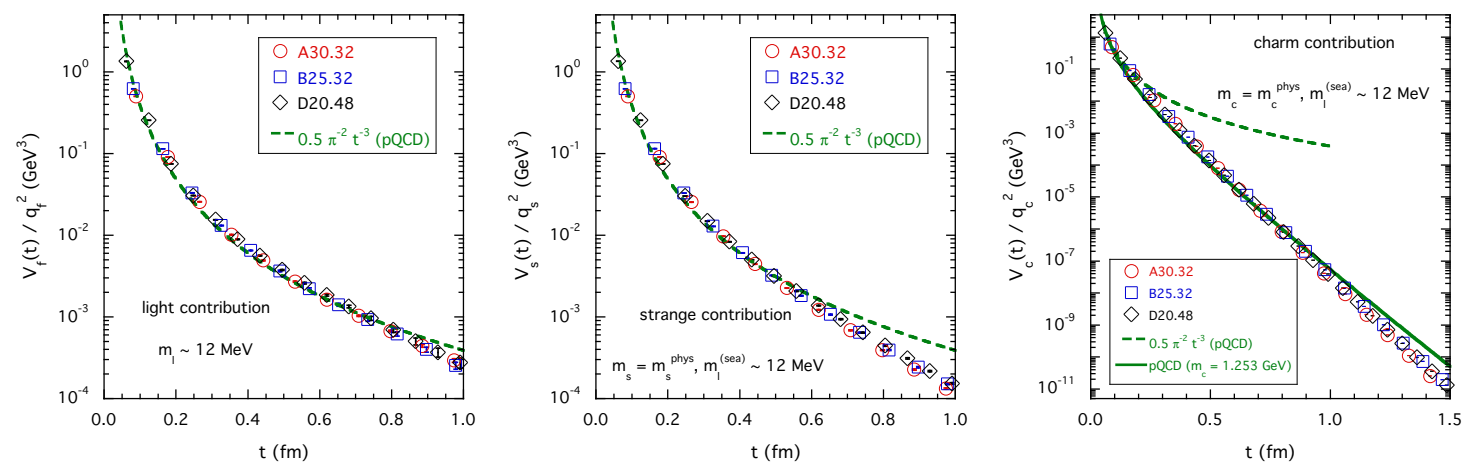

Figure 2. The vector correlator $V(t) / q_{f}^{2}$ (in physical units) in the case of the light (left panel), strange (middle panel) and charm (right panel) contributions for the ETMC gauge ensembles specified in the inset, which share an approximate common value of the light-quark mass $m_{\ell} \simeq 12 \mathrm{MeV}$ and differ in the values of the lattice spacing. The dashed lines represent the mass-indepedent pQCD prediction (3.22), while the solid line in the right panel is the pQCD prediction (3.21) calculated for an on-shell charm quark mass equal to $m_{c}=1.253 \mathrm{GeV}$.

Finally, it is interesting to estimate the contribution to $a_{\mu}^{\text {had }}$ coming from values of $Q^{2}$ larger than $Q_{\max }^{2} \simeq 1 / a^{2}$, which for our lattice setup is always larger than $4 \mathrm{GeV}^{2}$. Using the pQCD prediction (3.17) for the large $Q^{2}$-behavior of $\Pi_{R}\left(Q^{2}\right)$, one gets: $a_{\mu}^{\text {had }}\left(Q^{2}>4 \mathrm{GeV}^{2}\right) \simeq 1.3,0.11,0.06$ (in units of $\left.10^{-10}\right)$ in the case of the light, strange and charm contributions, respectively. The above findings represent only a small fraction of the uncertainties of the present lattice estimates of the three contributions to $a_{\mu}^{\text {had }}$ (see refs. $[13-15,19])$.

Alternatively we can check the change induced in the kernel function $\bar{f}(\bar{t})$ by cutting the upper integration limit in eq. (3.4) to $\omega_{\max }=Q_{\max } / m_{\mu} \simeq 1 /\left(a m_{\mu}\right)$. Since in our lattice setup $\omega_{\max } \gtrsim 20$, the kernel function $\bar{f}(\bar{t})$ changes at most by one part over $\simeq 10^{6}$ at small $\bar{t}$ in the case of the muon.

\subsection{Ground-state identification}

Our numerical simulations of the vector correlator $V(t)$ have been carried out in the context of a more general project aiming at the determination of the e.m. and strong IB corrections to pseudoscalar meson masses and leptonic decay constants [43]. In this project the bilinear operators were constructed adopting opposite values of the Wilson $r$-parameter. Thus, instead of eq. (3.5) the evaluation of the vector correlator has been carried out using the following local current:

$$
J_{\mu}(x)=Z_{A} q_{f} \bar{\psi}_{f^{\prime}}(x) \gamma_{\mu} \psi_{f}(x),
$$

where $\psi_{f^{\prime}}$ and $\psi_{f}$ represent two quarks with the same mass and charge, but regularized with opposite values of the Wilson $r$-parameter, i.e. $r_{f^{\prime}}=-r_{f}$. Being at maximal twist the current (3.23) renormalizes multiplicatively with the renormalization constant $Z_{A}$ of the axial current.

The choice (3.23) differs from the one given by eq. (3.5) by lattice artefacts of order $\mathcal{O}\left(a^{2}\right)$ and by the absence of disconnected insertions. The first point is illustrated in 


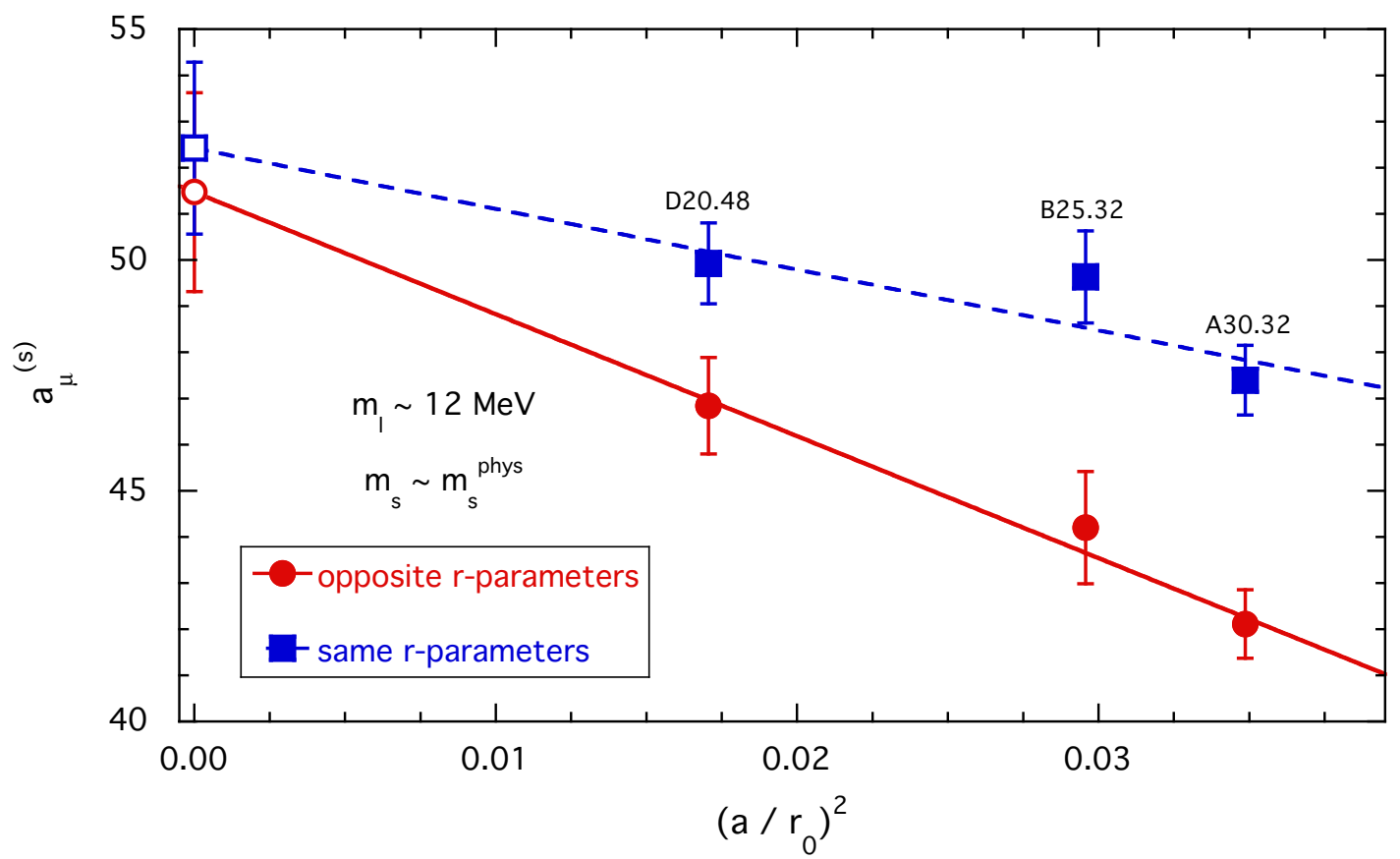

Figure 3. Results for the strange contribution to $a_{\mu}^{\text {had }}$ in units of $10^{-10}$ at lowest order versus the squared lattice spacing (in units of the Sommer parameter determined in ref. [34]), obtained using the local currents (3.5) (same r-parameters) and (3.23) (opposite r-parameters) for the ETMC gauge ensembles A30.32, B25.32 and D20.48, which share an approximate common value of the light-quark mass and differ by the values of the lattice spacing. The empty markers represent the value extrapolated in the continuum limit assuming a linear behavior in $a^{2}$.

figure 3, where the contribution of the strange quark to $a_{\mu}^{\text {had }}$, evaluated using either the current (3.23) or the connected insertion of eq. (3.5), is shown as a function of $a^{2}$ for the three ensembles A30.32, B25.32 and D20.48, which share an approximate common value of the light-quark mass. It can be seen that the same continuum limit is reached using either currents, confirming that the difference is due to discretization effects of order $\mathcal{O}\left(a^{2}\right)$. Moreover, the absence of disconnected insertions in the current (3.23) implies that the "purely connected" vector correlator based on the current (3.5) is a well defined quantity and admits the hadron decomposition necessary for having the representation (3.3) (see also refs. $[17,18]$ and therein quoted).

The statistical accuracy of the meson correlators is based on the use of the so-called "one-end" stochastic method [44], which includes spatial stochastic sources at a single time slice chosen randomly. Four stochastic sources (diagonal in the spin variable and dense in the color one) were adopted at first per each gauge configuration.

In the case of the light-quark contribution the signal-to-noise ratio does not allow to determine the ground-state mass $\bar{M}_{V}$ and the corresponding matrix element $\bar{Z}_{V}$ from the behavior of the vector correlator at large time distances. This is at variance with the case of the strange and charm contributions, as it is illustrated in figure 4, where it is also shown that discretization effects are sub-leading. 

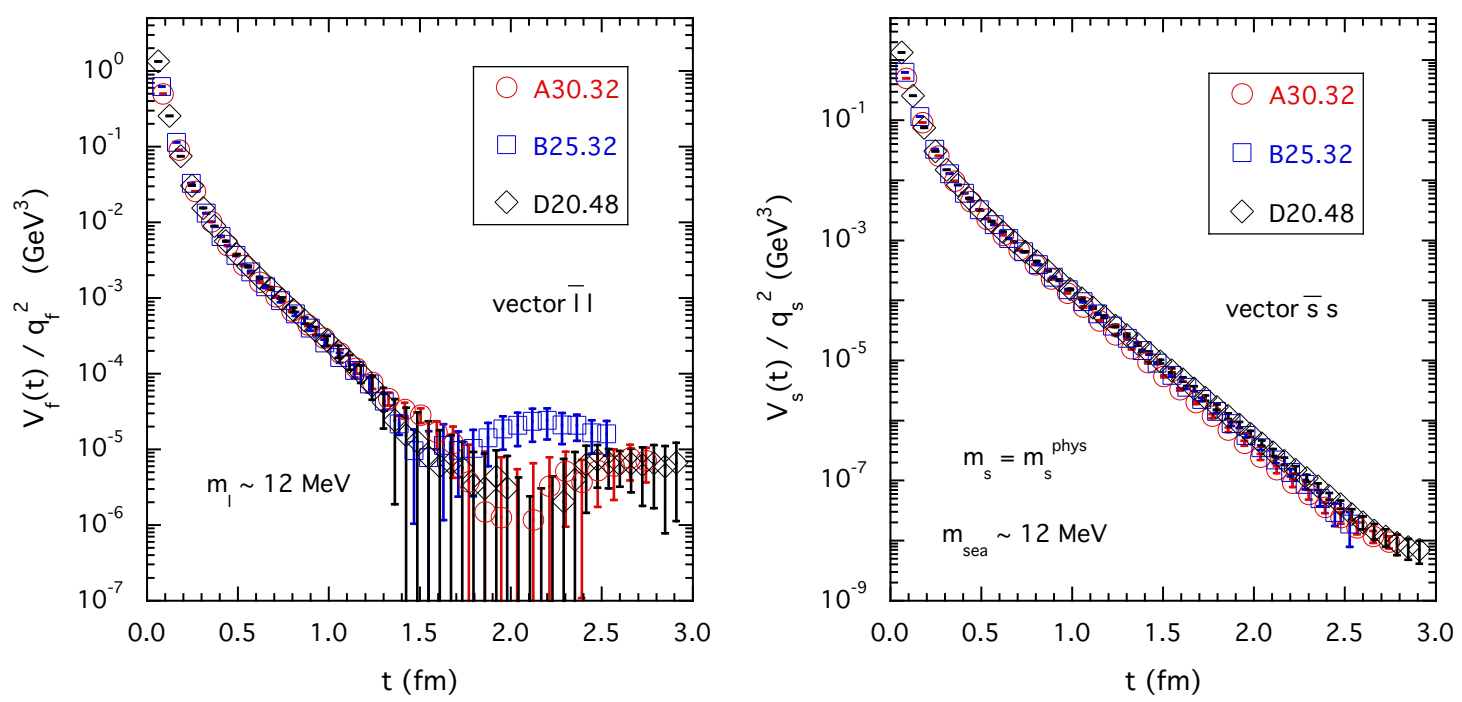

Figure 4. The vector correlator $V(t) / q_{f}^{2}$ (in physical units) in the case of the light (left panel) and strange (right panel) quarks for the ETMC gauge ensembles specified in the inset, which share an approximate common value of the light-quark mass $m_{\ell} \simeq 12 \mathrm{MeV}$ and differ in the values of the lattice spacing.

\begin{tabular}{|c|c|c|c|c|c|}
\hline$\beta$ & $V / a^{4}$ & $\bar{t}_{\min }(\bar{s} s)$ & $\bar{t}_{\max }(\bar{s} s)$ & $\bar{t}_{\min }(\bar{c} c)$ & $\bar{t}_{\max }(\bar{c} c)$ \\
\hline \multirow{3}{*}{1.90} & $32^{3} \times 64$ & 14 & 28 & 16 & 30 \\
\cline { 2 - 6 } & $24^{3} \times 48$ & 14 & 20 & 16 & 22 \\
\cline { 2 - 6 } & $20^{3} \times 48$ & 14 & 20 & 16 & 22 \\
\hline \multirow{2}{*}{1.95} & $32^{3} \times 64$ & 15 & 28 & 17 & 30 \\
\cline { 2 - 6 } & $24^{3} \times 48$ & 15 & 20 & 17 & 22 \\
\hline \multirow{2}{*}{2.10} & $48^{3} \times 96$ & 20 & 40 & 22 & 44 \\
\hline
\end{tabular}

Table 2. Values of $\bar{t}_{\min }$ and $\bar{t}_{\max }$ chosen to extract the ground-state signal from the strange and charm contributions to the vector correlator $\bar{V}(\bar{t})$ for the ETMC gauge ensembles of table 1 .

Thus, the identification of the ground-state is presently possible only in the case of $\bar{s} s$ and $\bar{c} c$ vector mesons. To improve the statistics we took a significative advantage by using the DD $-\alpha \mathrm{AMG}$ solver [45], which has allowed us to increase by a factor of 5 the number of stochastic sources in the case of the strange quark. In this way we find that the quality of the plateaux, shown in figure 5, is acceptable in the strange sector and nice in the charm one. In the case of the light-quark contribution an increase of the statistics by a factor $\approx 20$ is expected to be needed.

For each gauge ensemble the masses $\bar{M}_{V}$ and the matrix elements $\bar{Z}_{V}$ are extracted from a single exponential fit (including the proper backward signal) in the range $\bar{t}_{\text {min }} \leq \bar{t} \leq \bar{t}_{\text {max }}$. The values chosen for $\bar{t}_{\text {min }}$ and $\bar{t}_{\text {max }}$ are collected in table 2 . 

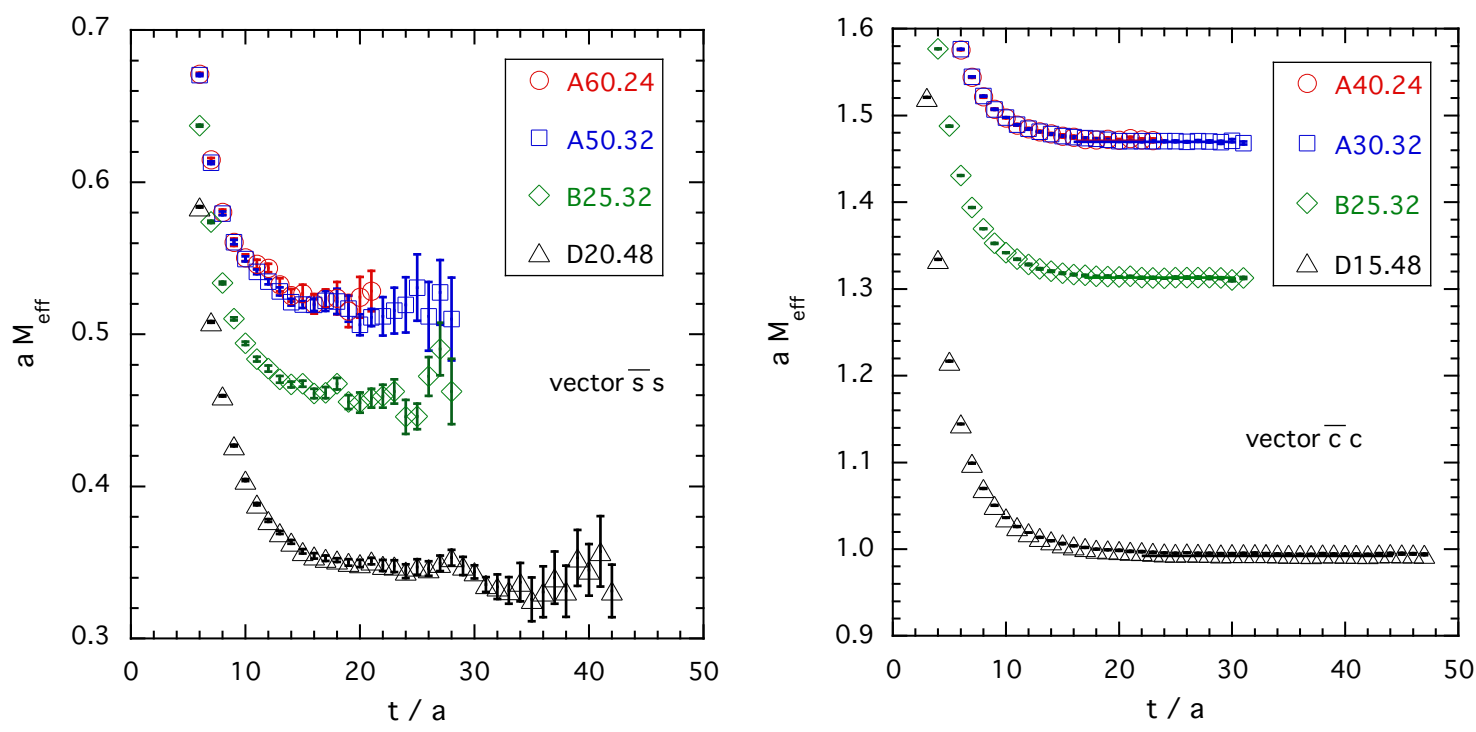

Figure 5. Effective mass of the vector correlator $\bar{V}(\bar{t})$ in the case of the strange (left panel) and charm (right panel) contributions for the ETMC gauge ensembles specified in the insets.

\section{Strange and charm contributions: lowest order}

Let's start by considering the evaluation of $a_{\mu}^{\text {had }}(<)$ and $a_{\mu}^{\text {had }}(>)$ defined in eqs. (3.2)-(3.3) for various values of the "cut" $\bar{T}_{\text {data }}$ chosen in the range between $\bar{t}_{\min }$ and $\bar{t}_{\text {max }}$ given in table 2 .

The results for the strange contribution to $a_{\mu}^{\text {had }}(<), a_{\mu}^{\text {had }}(>)$ and their sum $a_{\mu}^{\text {had }}$ obtained adopting four choices of $\bar{T}_{\text {data }}$, namely: $\bar{T}_{\text {data }}=\left(\bar{t}_{\min }+2\right),\left(\bar{t}_{\min }+\bar{t}_{\max }\right) / 2,\left(\bar{t}_{\max }-2\right)$ and $(\bar{T} / 2-4)$, are collected in table 3 for illustrative purposes in the case of few ETMC gauge ensembles.

The separation between $a_{\mu}^{\text {had }}(<)$ and $a_{\mu}^{\text {had }}(>)$ depends on the specific value of $\bar{T}_{\text {data }}$, as it should be, but their sum $a_{\mu}^{\text {had }}$ is almost independent of the choice of the value of $\bar{T}_{\text {data }}$ in the range between $\bar{t}_{\text {min }}$ and $\bar{t}_{\text {max }}$. This is also reassuring of the fact that the value of $a_{\mu}^{\text {had }}$ is not contaminated significantly by the presence of backward signals in the correlator $\bar{V}(\bar{t})$.

In the case of the charm contribution the value of $a_{\mu}^{\text {had }}(>)$ is always several orders of magnitude smaller than $a_{\mu}^{\text {had }}(<)$ and the latter turns out to be the same for all the four choices of $\bar{T}_{\text {data. }}$.

Note that for $\bar{T}_{\text {data }}=\bar{T} / 2-4$ the contribution $a_{\mu}^{\text {had }}(>)$, which depends on the analytic representation (3.3), does not exceed $\simeq 1.2 \%$ of the total value $a_{\mu}^{\text {had }}$ even at the smallest value of the time extension $\bar{T}$.

In what follows all the four choices of $\bar{T}_{\text {data }}$ will be employed in the various branches of our bootstrap analysis. The corresponding systematics is largely sub-dominant with respect to the other sources of uncertainties and it will not be given separately in the error budget.

The results obtained for the strange and charm contributions to $a_{\mu}^{\text {had }}$ are shown by the empty markers in figure 6 . We observe a mild dependence on the light-quark mass, being driven only by sea quarks, and also small residual FSEs visible only in the case of the 
ensemble A40.24

\begin{tabular}{|c|c|c|c|c|}
\hline $\bar{s} s$ & $\left(\bar{t}_{\min }+2\right)$ & $\left(\bar{t}_{\min }+\bar{t}_{\max }\right) / 2$ & $\left(\bar{t}_{\max }-2\right)$ & $(\bar{T} / 2-4)$ \\
\hline$a_{\mu}^{\text {had }}(<)$ & $38.03(28)$ & $38.65(29)$ & $39.10(29)$ & $39.67(30)$ \\
\hline$a_{\mu}^{\text {had }}(>)$ & $1.97(13)$ & $1.41(10)$ & $1.00(8)$ & $0.49(5)$ \\
\hline$a_{\mu}^{\text {had }}$ & $40.00(32)$ & $40.06(31)$ & $40.10(31)$ & $40.16(31)$ \\
\hline
\end{tabular}

ensemble A30.32

\begin{tabular}{|c|c|c|c|c|}
\hline $\bar{s} s$ & $\left(\bar{t}_{\min }+2\right)$ & $\left(\bar{t}_{\min }+\bar{t}_{\max }\right) / 2$ & $\left(\bar{t}_{\max }-2\right)$ & $(\bar{T} / 2-4)$ \\
\hline$a_{\mu}^{\text {had }}(<)$ & $40.44(19)$ & $42.77(23)$ & $43.26(25)$ & $43.32(25)$ \\
\hline$a_{\mu}^{\text {had }}(>)$ & $3.15(18)$ & $0.63(5)$ & $0.11(1)$ & $0.05(1)$ \\
\hline$a_{\mu}^{\text {had }}$ & $43.59(30)$ & $43.40(25)$ & $43.37(25)$ & $43.37(25)$ \\
\hline
\end{tabular}

ensemble B25.32

\begin{tabular}{|c|c|c|c|c|}
\hline $\bar{s} s$ & $\left(\bar{t}_{\min }+2\right)$ & $\left(\bar{t}_{\min }+\bar{t}_{\max }\right) / 2$ & $\left(\bar{t}_{\max }-2\right)$ & $(\bar{T} / 2-4)$ \\
\hline$a_{\mu}^{\text {had }}(<)$ & $40.83(14)$ & $43.18(17)$ & $44.05(18)$ & $44.16(19)$ \\
\hline$a_{\mu}^{\text {had }}(>)$ & $3.52(14)$ & $1.11(6)$ & $0.23(1)$ & $0.11(1)$ \\
\hline$a_{\mu}^{\text {had }}$ & $44.35(22)$ & $44.29(19)$ & $44.28(19)$ & $44.27(19)$ \\
\hline
\end{tabular}

ensemble D15.48

\begin{tabular}{|c|c|c|c|c|}
\hline $\bar{s} s$ & $\left(\bar{t}_{\min }+2\right)$ & $\left(\bar{t}_{\min }+\bar{t}_{\max }\right) / 2$ & $\left(\bar{t}_{\max }-2\right)$ & $(\bar{T} / 2-4)$ \\
\hline$a_{\mu}^{\mathrm{had}}(<)$ & $42.34(17)$ & $45.86(19)$ & $46.50(20)$ & $46.58(20)$ \\
\hline$a_{\mu}^{\text {had }}(>)$ & $4.27(18)$ & $0.75(5)$ & $0.10(1)$ & $0.02(1)$ \\
\hline$a_{\mu}^{\text {had }}$ & $46.61(24)$ & $46.61(20)$ & $46.60(20)$ & $46.60(20)$ \\
\hline
\end{tabular}

Table 3. Results for the strange contribution to $a_{\mu}^{\text {had }}(<), a_{\mu}^{\text {had }}(>)$ and their sum $a_{\mu}^{\text {had }}$, in units of $10^{-10}$, obtained assuming $\bar{T}_{\text {data }}=\left(\bar{t}_{\min }+2\right),\left(\bar{t}_{\min }+\bar{t}_{\max }\right) / 2,\left(\bar{t}_{\max }-2\right)$ and $(\bar{T} / 2-4)$ for the ETMC gauge ensembles A40.24, A30.32, B25.32 and D15.48. Errors are statistical only.

strange contribution. The errors of the data turn out to be dominated by the uncertainties of the scale setting, which are similar for all the gauge ensembles used in this work.

In ref. [13] a modification of the calculated $a_{\mu}^{\text {had }}$ at pion masses above the physical point has been proposed in order to weaken the pion mass dependence of the resulting $a_{\mu}^{\text {had }}$ for improving the reliability of the chiral extrapolation. Though the procedure of ref. [13] has been conceived mainly for the light contribution to $a_{\mu}^{\text {had }}$, we have explored its usefulness also in the case of the strange and charm contributions. The proposal consists in multiplying the Euclidean 4-momentum transfer $Q^{2}$ by a factor equal to $\left(M_{V} / M_{V}^{\text {phys }}\right)^{2}$ in order to modify the $Q^{2}$-dependence of the HVP function $\Pi_{R}\left(Q^{2}\right)$ without modifying its value at the physical point. One obtains the same effect in our master formulae by redefining the lepton mass as

$$
\bar{m}_{\mu}^{\mathrm{ELM}}=\bar{M}_{V} \frac{m_{\mu}}{M_{V}^{\mathrm{phys}}} .
$$



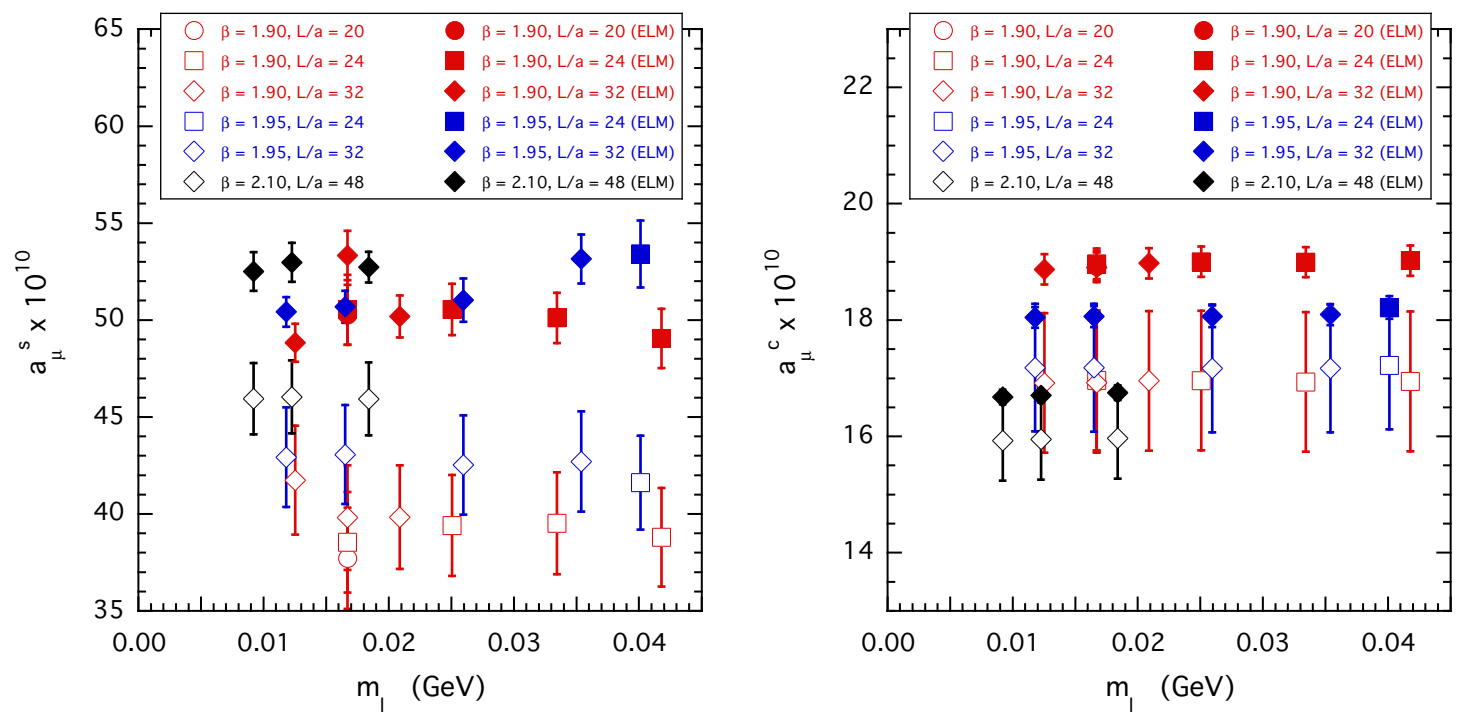

Figure 6. Results for the strange (left panel) and charm (right panel) contributions to $a_{\mu}^{\text {had }}$ in units of $10^{-10}$, evaluated with (filled markers) and without (empty markers) the ELM procedure described by eq. (4.1). The PDG values $M_{V}^{\text {(phys) }}=1.0195$ and $3.0969 \mathrm{GeV}$ [2] have been adopted for the physical $\bar{s} s$ and $\bar{c} c$ vector meson masses, respectively.

The expected advantage of the use of the effective lepton mass (4.1) comes from the fact that the kernel function, and therefore $a_{\mu}^{\text {had }}$, depends only on the lepton mass in lattice units (see eq. (3.4)). Thanks to eq. (4.1), which will be referred to as the Effective Lepton Mass (ELM) procedure, the knowledge of the value of the lattice spacing is not required and therefore the resulting $a_{\mu}^{\text {had }}$ is not affected by the uncertainties of the scale setting. The drawback of the ELM procedure is instead represented by its potential sensitivity to the statistical fluctuations of the vector meson mass extracted from the lattice data.

The results obtained adopting the ELM procedure (4.1) in the case of the strange and charm contributions to $a_{\mu}^{\text {had }}$ are shown by the filled markers in figure 6 , where the physical values for the $\bar{s} s$ and $\bar{c} c$ vector masses have been taken from PDG [2] (namely, $M_{V}^{\text {(phys) }}=1.0195$ and $3.0969 \mathrm{GeV}$, respectively). ${ }^{1}$ It can be seen that the ELM procedure reduces remarkably the overall uncertainty of the data. Moreover, it further weakens the pion mass dependence (in any case driven only by the sea quarks) and modifies the discretization effects, leading to a better scaling behavior of the data in the case of the charm contribution. Since the pion mass dependence is in any case quite mild, the ELM procedure can be viewed as an alternative way to perform the continuum extrapolation and to avoid the scale setting uncertainties.

Using the data obtained either with or without the ELM procedure we have performed a combined fit for the extrapolation to the physical pion mass, the continuum and infinite

\footnotetext{
${ }^{1}$ We have checked that the chiral and continuum extrapolations of the simulated vector meson masses are consistent with the PDG values within lattice uncertainties, which are dominated by the error of the lattice scale.
} 
volume limits using the following simple Ansatz

$$
a_{\mu}^{s, c}=A_{0}^{s, c}\left[1+A_{1}^{s, c} \xi+D^{s, c} a^{2}+F^{s, c} \xi \frac{e^{-M_{\pi} L}}{M_{\pi} L}\right],
$$

where $\xi \equiv M_{\pi}^{2} /\left(4 \pi f_{0}\right)^{2}$ and the exponential term is a phenomenological representation of possible finite size effects (FSEs). The results of the linear fit (4.2) are shown in figure 7 by the solid lines. In our combined fit the values of the parameters are determined by a $\chi^{2}$-minimization procedure adopting an uncorrelated $\chi^{2}$. The uncertainties on the fitting parameters do not depend on the value of the uncorrelated $\chi^{2}$, because they are obtained using the bootstrap procedure of ref. [34] (see section 3.1). This guarantees that all correlations among the lattice data points and among the fitting parameters are properly taken into account.

Averaging over the results corresponding to different fitting functions of the data either with or without the ELM procedure we get at the physical point

$$
\begin{aligned}
a_{\mu}^{s, \text { phys }} & =\left(53.1 \pm 1.6_{\text {stat }+ \text { fit }} \pm 1.5_{\text {input }} \pm 1.3_{\text {disc }} \pm 0.2_{\mathrm{FSE}} \pm 0.1_{\text {chir }}\right) \cdot 10^{-10} \\
& =\left(53.1 \pm 1.6_{\text {stat }+ \text { fit }} \pm 2.0_{\text {syst }}\right) \cdot 10^{-10}, \\
& =(53.1 \pm 2.5) \cdot 10^{-10},
\end{aligned}
$$

where

- ()$_{\text {stat+fit }}$ indicates the uncertainty induced by both the statistical errors and the fitting procedure itself;

- ()input is the error coming from the uncertainties of the input parameters of the eight branches of the quark mass analysis of ref. [34];

- ()$_{\text {disc }}$ is the uncertainty due to both discretization effects and scale setting, estimated by comparing the results obtained with and without the ELM procedure (4.1);

- ()$_{\mathrm{FSE}}$ is the error coming from including $\left(F^{s} \neq 0\right)$ or excluding $\left(F^{s}=0\right)$ the FSE correction. When FSEs are not included, all the gauge ensembles with $L / a=20$ and 24 are also not included;

- ()$_{\text {chir }}$ is the error coming from including $\left(A_{1}^{s} \neq 0\right)$ or excluding $\left(A_{1}^{s}=0\right)$ the linear term in the light-quark mass.

Our result (4.3) compares well with the $N_{f}=2+1+1$ result $a_{\mu}^{s, \text { phys }}=(53.41 \pm 0.59)$. $10^{-10}$ from the HPQCD collaboration [14], the $N_{f}=2+1$ finding $a_{\mu}^{s, \text { phys }}=\left(53.1 \pm 0.9_{-0.3}^{+0.1}\right)$. $10^{-10}$ obtained by the RBC/UKQCD collaboration [19], and with the recent $N_{f}=2$ result $a_{\mu}^{s, \text { phys }}=(51.1 \pm 1.7 \pm 0.4) \cdot 10^{-10}$ of ref. [21].

In the case of the charm contribution we obtain

$$
\begin{aligned}
a_{\mu}^{c, \text { phys }} & =\left(14.75 \pm 0.42_{\text {stat }+ \text { fit }} \pm 0.36_{\text {input }} \pm 0.10_{\text {disc }} \pm 0.03_{\mathrm{FSE}} \pm 0.01_{\text {chir }}\right) \cdot 10^{-10} \\
& =\left(14.75 \pm 0.42_{\text {stat }+ \text { fit }} \pm 0.37_{\text {syst }}\right) \cdot 10^{-10} \\
& =(14.75 \pm 0.56) \cdot 10^{-10}
\end{aligned}
$$



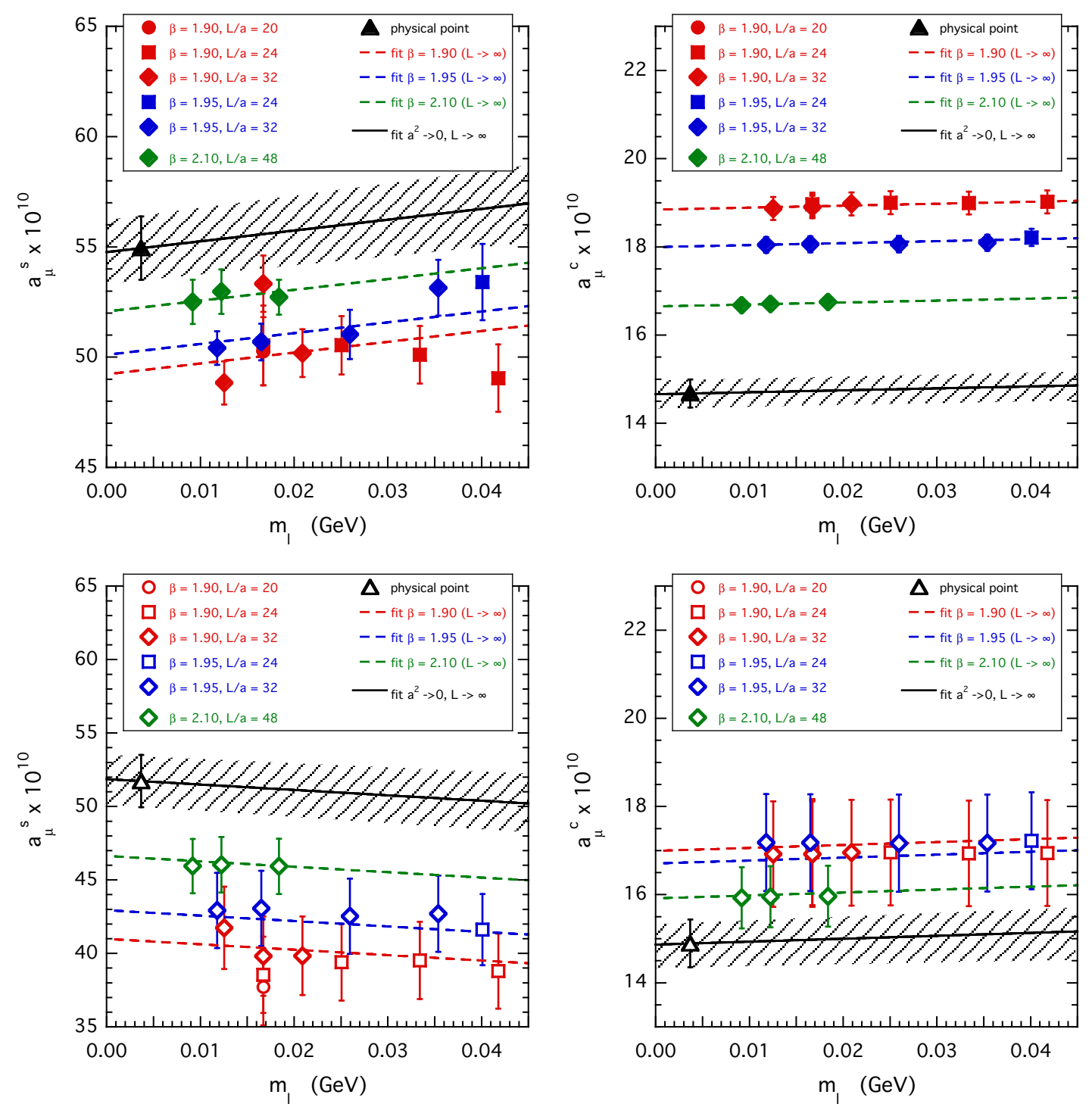

Figure 7. Results for the strange (left panels) and charm (right panels) contributions to $a_{\mu}^{\text {had }}$ in units of $10^{-10}$ for the ETMC gauge ensembles of table 1. Upper (lower) panels correspond to the data obtained with (without) the ELM procedure (4.1). The dashed lines correspond to the linear fit (4.2) including the discretization term in the infinite volume limit. The solid lines correspond to the linear fit (4.2) in the continuum and infinite volume limits, while the shaded areas identify the corresponding uncertainty at the level of one standard deviation. The triangles are the results of the extrapolation at the physical pion mass and in the continuum and infinite volume limits based on the linear fit (4.2).

where the errors are estimated as in the case of the strange quark contribution. Our finding (4.4) agrees with the $N_{f}=2+1+1$ result $a_{\mu}^{c \text {,phys }}=(14.42 \pm 0.39) \cdot 10^{-10}$ from the HPQCD collaboration [14] and with recent $N_{f}=2$ one $a_{\mu}^{c \text {,phys }}=(14.3 \pm 0.2 \pm 0.1) \cdot 10^{-10}$ of ref. [21]. 


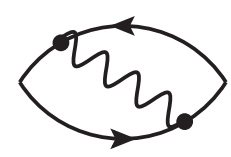

(a)

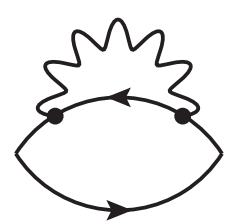

(b)

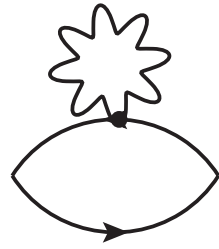

(c)

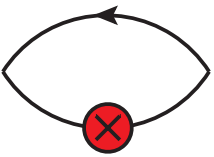

(d)

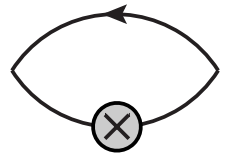

(e)

Figure 8. Fermionic connected diagrams contributing to the e.m. corrections to $a_{\mu}^{\text {had }}$ exchange (a), self energy (b), tadpole (c), pseudoscalar (d) and scalar (e) insertions. Solid lines represent quark propagators.

\section{Strange and charm contributions: e.m. corrections}

Let's now turn to the e.m. corrections at leading order in $\alpha_{\mathrm{em}}$ to $a_{\mu}^{\text {had }}$, which using the expansion method of ref. [28] require the evaluation of the self-energy, exchange, tadpole, pseudoscalar and scalar insertion diagrams depicted in figure 8 .

For each quark flavor $f$ the e.m. correction $\delta V(t)$ to the vector correlator is given by

$$
\delta V(t) \equiv \delta V^{\text {self }}(t)+\delta V^{\text {exch }}(t)+\delta V^{\mathrm{tad}}(t)+\delta V^{\mathrm{PS}}(t)+\delta V^{S}(t)
$$

with

$$
\begin{aligned}
\delta V^{\text {self }+ \text { exch }}(t) & =\frac{4 \pi \alpha_{\mathrm{em}}}{3} \sum_{i=1,2,3} \sum_{\vec{x}, y_{1}, y_{2}}\left\langle 0\left|T\left\{J_{i}^{\dagger}(\vec{x}, t) \sum_{\mu} J_{\mu}^{C}\left(y_{1}\right) J_{\mu}^{C}\left(y_{2}\right) J_{i}(0)\right\}\right| 0\right\rangle, \\
\delta V^{\mathrm{tad}}(t) & =\frac{4 \pi \alpha_{\mathrm{em}}}{3} \sum_{i=1,2,3} \sum_{\vec{x}, y}\left\langle 0\left|T\left\{J_{i}^{\dagger}(\vec{x}, t) \sum_{\nu} T_{\nu}(y) J_{i}(0)\right\}\right| 0\right\rangle \\
\delta V^{\mathrm{PS}}(t) & =\frac{2 \delta m_{f}^{\text {crit }}}{3} \sum_{i=1,2,3} \sum_{\vec{x}, y}\left\langle 0\left|T\left\{J_{i}^{\dagger}(\vec{x}, t) i \bar{\psi}_{f}(y) \gamma_{5} \psi_{f}(y) J_{i}(0)\right\}\right| 0\right\rangle \\
\delta V^{S}(t) & =-\frac{2 m_{f}}{3 Z_{m} \mathcal{Z}_{f}} \sum_{i=1,2,3} \sum_{\vec{x}, y}\left\langle 0\left|T\left\{J_{i}^{\dagger}(\vec{x}, t) \bar{\psi}_{f}(y) \psi_{f}(y) J_{i}(0)\right\}\right| 0\right\rangle
\end{aligned}
$$

where $J_{\mu}^{C}(y)$ and $T_{\nu}(y)$ are given in eqs. (3.6) and (3.8), respectively. In eq. (5.1) $\delta m_{f}^{\text {crit }}$ is the e.m. shift of the critical mass for the quark flavor $f$, while $Z_{m}$ and $\mathcal{Z}_{f}$ are related to the mass renormalization constants (RCs) in QCD and QCD+QED. For our maximally twisted-mass setup $\delta m_{f}^{\text {crit }}$ has been determined in ref. [29], while $1 / Z_{m}=Z_{P}$, where $Z_{P}$ is the $\mathrm{RC}$ of the pseudoscalar density evaluated in ref. [34]. For $1 / \mathcal{Z}_{f}$ we use the perturbative result at leading order in $\alpha_{\mathrm{em}}$ in the $\overline{\mathrm{MS}}$ scheme, given by $[46,47]$

$$
\frac{1}{\mathcal{Z}_{f}}(\overline{\mathrm{MS}}, \mu)=\frac{\alpha_{\mathrm{em}} q_{f}^{2}}{4 \pi}[6 \log (a \mu)-22.5954],
$$

where the renormalization scale $\mu$ is taken to be equal to $\mu=2 \mathrm{GeV}$, at which we consider that the renormalized quark masses in QCD and QCD+QED coincide (see ref. [29]). 
The removal of the photon zero-mode is done according to $\mathrm{QED}_{L}$ [48], i.e. the photon field $A_{\mu}$ satisfies $A_{\mu}\left(k_{0}, \vec{k}=\overrightarrow{0}\right) \equiv 0$ for all $k_{0}$.

Within the quenched QED approximation, which neglects the effects of the sea-quark electric charges, the correlator $\delta V^{\text {self }}(t)+\delta V^{\text {exch }}(t)$ corresponds to the sum of the diagrams $8 \mathrm{a}-8 \mathrm{~b}$, while the correlators $\delta V^{\mathrm{tad}}(t), \delta V^{\mathrm{PS}}(t)$ and $\delta V^{S}(t)$ represent the contributions of the diagrams $8 \mathrm{c}, 8 \mathrm{~d}$ and $8 \mathrm{e}$, respectively. In the quenched QED approximation the shift $\delta m_{f}^{\text {crit }}$ is proportional to $\alpha_{\mathrm{em}} q_{f}^{2}$ (see for details ref. [29]).

In addition one has to consider also the QED contribution to the renormalization constant of the vector current (3.23), namely

$$
Z_{A}=Z_{A}^{(0)}+\delta Z_{A}+\mathcal{O}\left(\alpha_{\mathrm{em}}^{2}\right)
$$

where $Z_{A}^{(0)}$ is the renormalization constant (RC) of the current in absence of QED (determined in ref. [34]) and $\delta Z_{A}$ is the $\mathcal{O}\left(\alpha_{\mathrm{em}}\right) \mathrm{RC}$. The latter can be written as

$$
\delta Z_{A}=Z_{A}^{(0)} \cdot Z_{A}^{(\mathrm{em})} \cdot Z_{A}^{(\mathrm{fact})},
$$

where $Z_{A}^{(\mathrm{em})}$ is the one-loop perturbative estimate of the QED effect at order $\mathcal{O}\left(\alpha_{s}^{0}\right)$ in the strong coupling and $Z_{A}^{(\text {fact })}$ takes into account corrections of order $\mathcal{O}\left(\alpha_{\mathrm{em}} \alpha_{s}^{n}\right)$ with $n \geq 1$, i.e. corrections to the "naive factorization" approximation in which $Z_{A}^{(\text {fact })}=1$. In the appendix A we present our non-perturbative estimate $Z_{A}^{\text {(fact) }}=0.9 \pm 0.1$, obtained through the use of the axial Ward-Takahashi identity (WTI) derived in the presence of QED effects. ${ }^{2}$ Using the result $Z_{A}^{(\mathrm{em})}=-15.7963 \alpha_{\mathrm{em}} q_{f}^{2} /(4 \pi)$ from refs. [46, 47], we have to add to eq. (5.1) the following contribution

$$
\delta V^{Z_{A}}(t) \equiv-2.51406 \alpha_{\mathrm{em}} q_{f}^{2} Z_{A}^{(\mathrm{fact})} V(t) .
$$

Thus, the e.m. corrections $\delta a_{\mu}^{\text {had }}$ can be written as

$$
\delta a_{\mu}^{\text {had }} \equiv \delta a_{\mu}^{\text {had }}(<)+\delta a_{\mu}^{\text {had }}(>)
$$

with (see eqs. (3.2)-(3.3))

$$
\begin{aligned}
\delta a_{\mu}^{\text {had }}(<) & =4 \alpha_{\mathrm{em}}^{2} \sum_{\bar{t}=0}^{\bar{T}_{\text {data }}} \bar{f}(\bar{t}) \delta \bar{V}(\bar{t}) \\
\delta a_{\mu}^{\text {had }}(>) & =4 \alpha_{\mathrm{em}}^{2} \sum_{\bar{t}=\bar{T}_{\text {data }}+1}^{\infty} \bar{f}(\bar{t}) \delta\left[\frac{\bar{Z}_{V}}{2 \bar{M}_{V}} e^{-\bar{M}_{V} \bar{t}}\right] \\
& =4 \alpha_{\mathrm{em}}^{2} \sum_{\bar{t}=\bar{T}_{\text {data }}+1}^{\infty} \bar{f}(\bar{t}) \frac{\bar{Z}_{V}}{2 \bar{M}_{V}} e^{-\bar{M}_{V} \bar{t}}\left[\frac{\delta \bar{Z}_{V}}{\bar{Z}_{V}}-\frac{\delta \bar{M}_{V}}{\bar{M}_{V}}\left(1+\bar{M}_{V} \bar{t}\right)\right],
\end{aligned}
$$

where $\delta \bar{M}_{V}$ and $\delta \bar{Z}_{V}$ can be determined, respectively, from the "slope" and the "intercept" of the ratio $\delta \bar{V}(\bar{t}) / \bar{V}(\bar{t})$ at large time distances $\bar{t}_{\min } \leq \bar{t} \leq \bar{t}_{\max }$ (see refs. [27-29]). Note

\footnotetext{
${ }^{2} \mathrm{~A}$ different non-perturbative procedure for evaluating the QED contribution to the $\mathrm{RC}$ of the local vector current has been recently developed in ref. [32].
} 

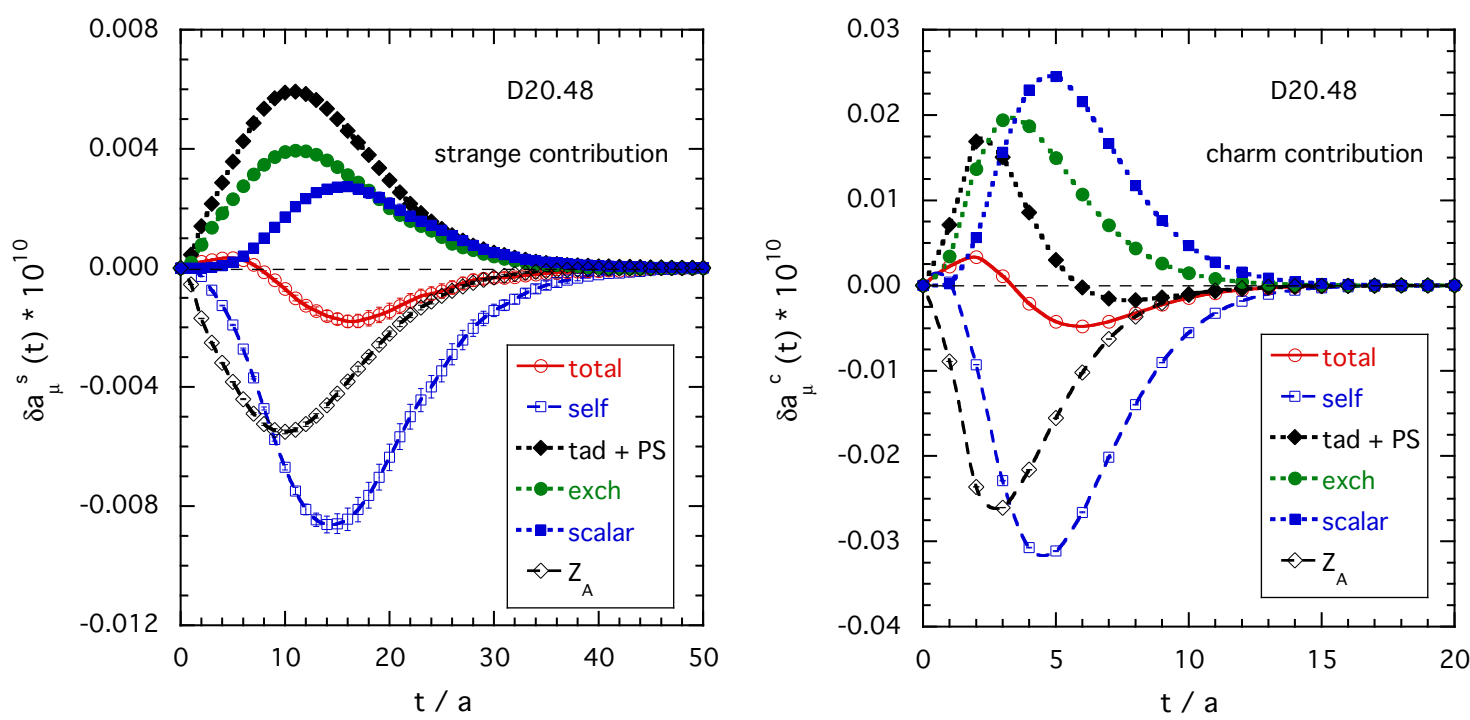

Figure 9. The time behavior of the integrand function $\delta a_{\mu}^{\text {had }}(t)$ in the r.h.s. of eqs. (5.11)-(5.12) in the case of the strange (left panel) and charm (right panel) quarks in units of $10^{-10}$, obtained for the ETMC gauge ensemble D20.48. The labels "self", "tad + PS", "exch", "scalar" and " $Z_{A}$ " indicate the contributions of the diagrams $8 \mathrm{~b}, 8 \mathrm{c}+8 \mathrm{~d}, 8 \mathrm{a}, 8 \mathrm{e}$ and the one generated by the QED effect in the $\mathrm{RC} Z_{A}$ of the local vector current at leading order in $\alpha_{\mathrm{em}}$ (see eq. (5.9)) with $Z_{A}^{\text {(fact) }}=0.9$. The label "total" corresponds to the sum of all the contributions.

that all the quantities $\delta \bar{V}, \delta \bar{Z}_{V}$ and $\delta \bar{M}_{V}$ are proportional to $\alpha_{\mathrm{em}} q_{f}^{2}$, which make $\delta a_{\mu}^{\text {had }}$ proportional to $\alpha_{\mathrm{em}}^{3} q_{f}^{4}$.

The time dependence of the integrand function in the r.h.s. of eqs. (5.11)-(5.12) is shown in figure 9 in the case of the ETMC gauge ensemble D20.48. The contributions coming from the various diagrams of figure 8 as well as from the additional term (5.9) are determined quite precisely and are characterized by different signs. Partial cancellations among the various contributions occur in the total sum, which turns out to be smaller than each individual contributions. Thus, even a $10 \%$ uncertainty on the $\mathrm{RC} \delta Z_{A}$ may have a larger impact on the final uncertainty of $\delta a_{\mu}^{\text {had }}$, as it will be shown later on.

The results for the strange contribution to $\delta a_{\mu}^{\text {had }}(<), \delta a_{\mu}^{\text {had }}(>)$ and their sum $\delta a_{\mu}^{\text {had }}$, obtained adopting the four choices of $\bar{T}_{\text {data }}$, namely: $\bar{T}_{\text {data }}=\left(\bar{t}_{\min }+2\right),\left(\bar{t}_{\min }+\bar{t}_{\max }\right) / 2$, $\left(\bar{t}_{\max }-2\right)$ and $(\bar{T} / 2-4)$, are collected in table 4 for some of the ETMC gauge ensembles.

As in the case of the lowest-order terms $a_{\mu}^{\text {had }}(<)$ and $a_{\mu}^{\text {had }}(>)$, we find that the separation between $\delta a_{\mu}^{\text {had }}(<)$ and $\delta a_{\mu}^{\text {had }}(>)$ depends on the specific value of $\bar{T}_{\text {data }}$, as it should be, but their sum $\delta a_{\mu}^{\text {had }}$ is largely independent of the choice of the value of $\bar{T}_{\text {data }}$ in the range between $\bar{t}_{\min }$ and $\bar{t}_{\max }$ within the statistical uncertainties. As in the case of the lowest-order term, the contribution $\delta a_{\mu}^{\text {had }}(>)$, which depends on the analytic representation (5.12), is significantly reduced at $\bar{T}_{\text {data }}=\bar{T} / 2-4$, where it does not exceed the statistical uncertainty of $\delta a_{\mu}^{\text {had }}$.

In the case of the charm contribution the value of $\delta a_{\mu}^{\text {had }}(>)$ is always several orders of magnitude smaller than $\delta a_{\mu}^{\text {had }}(<)$ and the latter turns out to be the same for all the four choices of $\bar{T}_{\text {data. }}$. 
ensemble A40.24

\begin{tabular}{|c|c|c|c|c|}
\hline $\bar{s} s$ & $\left(\bar{t}_{\min }+2\right)$ & $\left(\bar{t}_{\min }+\bar{t}_{\max }\right) / 2$ & $\left(\bar{t}_{\max }-2\right)$ & $(\bar{T} / 2-4)$ \\
\hline$\delta a_{\mu}^{\text {had }}(<)$ & $-1.26(13)$ & $-1.36(14)$ & $-1.45(14)$ & $-1.58(15)$ \\
\hline$\delta a_{\mu}^{\text {had }}(>)$ & $-0.40(7)$ & $-0.31(6)$ & $-0.24(5)$ & $-0.13(3)$ \\
\hline$\delta a_{\mu}^{\text {had }}$ & $-1.66(16)$ & $-1.67(16)$ & $-1.69(16)$ & $-1.71(16)$ \\
\hline
\end{tabular}

ensemble A30.32

\begin{tabular}{|c|c|c|c|c|}
\hline $\bar{s} s$ & $\left(\bar{t}_{\min }+2\right)$ & $\left(\bar{t}_{\min }+\bar{t}_{\max }\right) / 2$ & $\left(\bar{t}_{\max }-2\right)$ & $(\bar{T} / 2-4)$ \\
\hline$\delta a_{\mu}^{\text {had }}(<)$ & $-1.03(10)$ & $-1.44(15)$ & $-1.56(17)$ & $-1.58(17)$ \\
\hline$\delta a_{\mu}^{\text {had }}(>)$ & $-0.56(8)$ & $-0.16(3)$ & $-0.03(1)$ & $-0.02(1)$ \\
\hline$\delta a_{\mu}^{\text {had }}$ & $-1.59(18)$ & $-1.60(17)$ & $-1.59(17)$ & $-1.60(18)$ \\
\hline
\end{tabular}

ensemble B25.32

\begin{tabular}{|c|c|c|c|c|}
\hline $\bar{s} s$ & $\left(\bar{t}_{\min }+2\right)$ & $\left(\bar{t}_{\min }+\bar{t}_{\max }\right) / 2$ & $\left(\bar{t}_{\max }-2\right)$ & $(\bar{T} / 2-4)$ \\
\hline$\delta a_{\mu}^{\text {had }}(<)$ & $-1.35(12)$ & $-1.80(15)$ & $-2.05(17)$ & $-2.09(18)$ \\
\hline$\delta a_{\mu}^{\text {had }}(>)$ & $-0.80(8)$ & $-0.34(4)$ & $-0.09(1)$ & $-0.05(1)$ \\
\hline$\delta a_{\mu}^{\text {had }}$ & $-2.15(18)$ & $-2.14(18)$ & $-2.14(18)$ & $-2.14(18)$ \\
\hline
\end{tabular}

ensemble D15.48

\begin{tabular}{|c|c|c|c|c|}
\hline $\bar{s} s$ & $\left(\bar{t}_{\min }+2\right)$ & $\left(\bar{t}_{\min }+\bar{t}_{\max }\right) / 2$ & $\left(\bar{t}_{\max }-2\right)$ & $(\bar{T} / 2-4)$ \\
\hline$\delta a_{\mu}^{\mathrm{had}}(<)$ & $-1.27(9)$ & $-1.86(15)$ & $-2.02(18)$ & $-2.04(19)$ \\
\hline$\delta a_{\mu}^{\mathrm{had}}(>)$ & $-0.77(13)$ & $-0.19(4)$ & $-0.03(1)$ & $-0.01(1)$ \\
\hline$\delta a_{\mu}^{\mathrm{had}}$ & $-2.04(20)$ & $-2.05(19)$ & $-2.05(19)$ & $-2.05(19)$ \\
\hline
\end{tabular}

Table 4. Results for the strange contribution to $\delta a_{\mu}^{\text {had }}(<), \delta a_{\mu}^{\text {had }}(>)$ and their sum $\delta a_{\mu}^{\text {had }}$, in units of $10^{-12}$, obtained assuming $\bar{T}_{\text {data }}=\left(\bar{t}_{\min }+2\right),\left(\bar{t}_{\min }+\bar{t}_{\max }\right) / 2,\left(\bar{t}_{\max }-2\right)$ and $(\bar{T} / 2-4)$ for the ETMC gauge ensembles A40.24, A30.32, B25.32 and D15.48. Errors are statistical only.

The precision of the lattice data can be drastically improved by forming the ratio of the e.m. correction over the lowest-order term. Therefore, in what follows we perform our analysis of the ratio $\delta a_{\mu}^{\text {had }} / a_{\mu}^{\text {had }}$, which is shown in figure 10. We have checked that in the case of the e.m. corrections the use of the ELM procedure (4.1) does not improve the precision of the lattice data.

It can be seen from figure 10 that the dependence on the light-quark mass $m_{\ell}$ is quite mild, being driven only by sea quarks, and that the uncertainties of the data are dominated by the error on the $\mathrm{RC} \delta Z_{A}$, which has been taken to be the same for all the gauge ensembles used in this work (see appendix A).

The FSEs are visible only in the case of the strange quark. A theoretical calculation of FSEs for $\delta a_{\mu}^{\text {had }}$ is not yet available. According to the general findings of ref. [49] the universal FSEs are expected to vanish, since they depend on the global charge of the meson states appearing in the spectral decomposition of the vector correlator $\delta V(t)$. Moreover, 

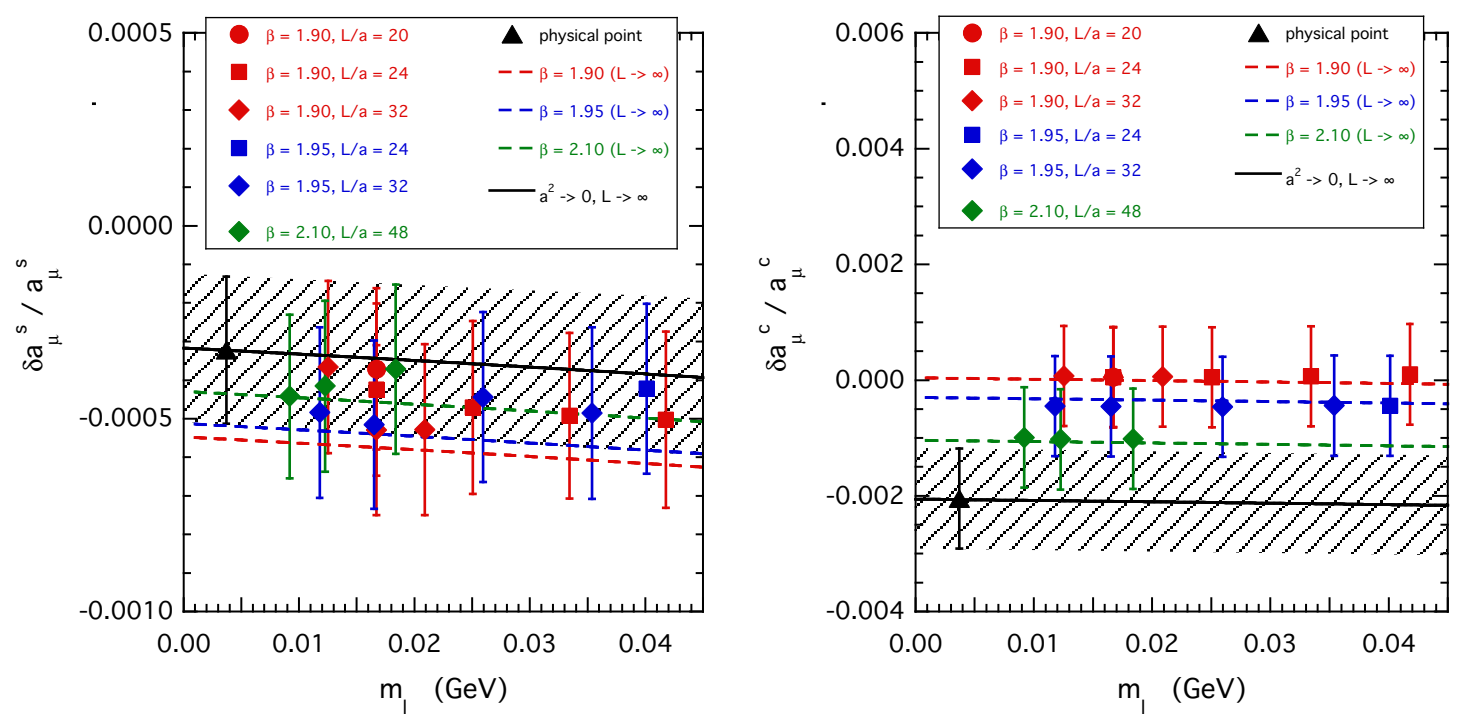

Figure 10. Results for the strange (left panel) and charm (right panel) contributions to $\delta a_{\mu}^{\text {had }} / a_{\mu}^{\text {had }}$, obtained for the ETMC gauge ensembles of table 1. The dashed lines correspond to the linear fit (5.13) including the discretization term in the infinite volume limit. The solid lines correspond to the linear fit in the continuum and infinite volume limits, while the shaded areas identify the corresponding uncertainty at the level of one standard deviation. The triangles are the results of the extrapolation at the physical pion mass and in the continuum and infinite volume limits. The data are evaluated without adopting the ELM procedure (4.1).

the structure-dependent (SD) FSEs are expected to start at order $\mathcal{O}\left(1 / L^{2}\right)$. According to the effective field theory approach of ref. [50], one might argue that in the case of mesons with vanishing charge radius (as the ones appearing in the correlator $\delta V(t)$ ) the SD FSEs may start at order $\mathcal{O}\left(1 / L^{3}\right)$. Therefore we adopt the following simple fitting function

$$
\frac{\delta a_{\mu}^{s, c}}{a_{\mu}^{s, c}}=\delta A_{0}^{s, c}+\delta A_{1}^{s, c} m_{\ell}+\delta D^{s, c} a^{2}+\delta F^{s, c} \frac{1}{L^{n}}
$$

where the power $n$ can be put equal to $n=2$ or $n=3$. In fitting our data we do not observe sensitivity to the above choices of the power $n$ within the statistical uncertainties.

At the physical pion mass and in the continuum and infinite volume limits we get

$$
\begin{aligned}
\frac{\delta a_{\mu}^{s, \text { phys }}}{a_{\mu}^{s, \text { phys }}} & =-0.000332(46)_{\text {stat }+ \text { fit }}(6)_{\text {input }}(8)_{\mathrm{FSE}}(4)_{\mathrm{chir}}(2)_{\mathrm{disc}}(208)_{Z_{A}}, \\
& =-0.000332(46)_{\text {stat }+\mathrm{fit}}(208)_{\mathrm{syst}}, \\
& =-0.000332(213),
\end{aligned}
$$

and

$$
\begin{aligned}
\frac{\delta a_{\mu}^{c, \text { phys }}}{a_{\mu}^{c \text { phys }}} & =-0.00205(12)_{\text {stat }+ \text { fit }}(1)_{\text {input }}(1)_{\mathrm{FSE}}(1)_{\mathrm{chir}}(1)_{\mathrm{disc}}(85)_{Z_{A}} \\
& =-0.00205(12)_{\mathrm{stat}+\mathrm{fit}}(85)_{\mathrm{syst}}, \\
& =-0.00205(86)
\end{aligned}
$$


where

- ()$_{\text {stat+fit }}$ indicates the uncertainty induced by both the statistical errors and the fitting procedure itself;

- ()input is the error coming from the uncertainties of the input parameters of the eight branches of the quark mass analysis of ref. [34];

- ()$_{\text {disc }}$ is the uncertainty due to both discretization effects and scale setting, estimated by comparing the results obtained with and without the ELM procedure (4.1);

- ()$_{\mathrm{FSE}}$ is the error coming from including $\left(\delta F^{s} \neq 0\right)$ or excluding $\left(\delta F^{s}=0\right)$ the FSE correction. When FSEs are not included, all the gauge ensembles with $L / a=24$ are also not included;

- ()$_{\text {chir }}$ is the error coming from including $\left(\delta A_{1}^{s} \neq 0\right)$ or excluding $\left(\delta A_{1}^{s}=0\right)$ the linear term in the light-quark mass.

- ()$_{Z_{A}}$ is the error generated by the uncertainty on the RC $Z_{A}^{f a c t}$ (see eq. (5.8)), which turns out to be by far the dominant source of uncertainty.

Using the lowest-order results (4.3)-(4.4) we obtain

$$
\begin{aligned}
& \delta a_{\mu}^{s, \text { phys }}=-0.018(11) \cdot 10^{-10}, \\
& \delta a_{\mu}^{c, \text { phys }}=-0.030(13) \cdot 10^{-10} .
\end{aligned}
$$

Thus, the e.m. corrections to $\delta a_{\mu}^{s}$ and $\delta a_{\mu}^{c}$ turn out to be negligible with respect to the current uncertainties of the lowest-order terms.

\section{Conclusions}

We have presented a lattice calculation of the HVP contribution of strange and charm quarks to the anomalous magnetic moment of the muon at orders $\mathcal{O}\left(\alpha_{\mathrm{em}}^{2}\right)$ and $\mathcal{O}\left(\alpha_{\mathrm{em}}^{3}\right)$ in the e.m. coupling.

We have employed the gauge configurations generated by the European Twisted Mass Collaboration with $N_{f}=2+1+1$ dynamical quarks at three values of the lattice spacing ( $a \simeq 0.062-0.089 \mathrm{fm})$ with pion masses in the range $M_{\pi} \simeq 210-450 \mathrm{MeV}$ and with strange and charm quark masses tuned at their physical values.

In this work we have taken into account only connected diagrams, in which each quark flavor contributes separately, and a direct summation of the relevant correlators over the Euclidean time distances has been performed, adopting the local lattice version of the e.m. current operator.

As for the calculation of the e.m. corrections in the strange and charm sectors, we have adopted the RM123 approach of ref. [28], based on the expansion of the lattice path-integral in powers of the small e.m. coupling, namely $\alpha_{\mathrm{em}} \approx 1 \%$. 
After extrapolation to the physical pion mass and to the continuum limit our results for $a_{\mu}^{\text {had }}$ are for the lowest-order contributions

$$
\begin{aligned}
& a_{\mu}^{s}\left(\alpha_{\mathrm{em}}^{2}\right)=(53.1 \pm 2.5) \cdot 10^{-10}, \\
& a_{\mu}^{c}\left(\alpha_{\mathrm{em}}^{2}\right)=(14.75 \pm 0.56) \cdot 10^{-10}
\end{aligned}
$$

and for the e.m. corrections

$$
\begin{aligned}
& a_{\mu}^{s}\left(\alpha_{\mathrm{em}}^{3}\right)=(-0.018 \pm 0.011) \cdot 10^{-10}, \\
& a_{\mu}^{c}\left(\alpha_{\mathrm{em}}^{3}\right)=(-0.030 \pm 0.013) \cdot 10^{-10},
\end{aligned}
$$

which show that the latter ones are negligible with respect to the present uncertainties of the lowest-order terms. We stress that the current uncertainties on the e.m. corrections $\delta a_{\mu}^{s}$ and $\delta a_{\mu}^{c}$ are of the order of $\sim 60 \%$ and $\sim 40 \%$, since they are dominated by the uncertainty on the $\mathrm{RC} Z_{A}$ of the local vector current, which has been estimated through the axial Ward-Takahashi identity (WTI) derived in the presence of QED effects (see appendix A). A dedicated study aimed at the determination of the RCs of bilinear operators in presence of QED employing non-perturbative renormalization schemes, like the RI-MOM one, is expected to improve significantly the precision of the calculation of the e.m. corrections and isospin-breaking effects on $a_{\mu}^{\text {had }}$.

Our findings demonstrate that the expansion method of ref. [28], which has been already applied successfully to the calculation of e.m. corrections to meson masses [28, 29] and to the leptonic decays of pions and kaons [30,31], works as well also in the case of the HVP contribution to the muon anomalous magnetic moment. The application of the approach presented in this work to the case of the $u$ - and $d$-quark contributions is ongoing.

\section{Acknowledgments}

We warmly thank our colleagues of the ETM collaboration, in particular R. Frezzotti, K. Jansen, M. Petschlies, G.C. Rossi, N. Tantalo and C. Tarantino, for many fruitful discussions and comments. We gratefully acknowledge the CPU time provided by PRACE under the project Pra10-2693 "QED corrections to meson decay rates in Lattice QCD" and by CINECA under the specific initiative INFN-LQCD123 on the BG/Q system Fermi at CINECA (Italy). V.L., G.M., S.S. thank MIUR (Italy) for partial support under the contract PRIN 2015. G.M. also acknowledges partial support from ERC Ideas Advanced Grant n. 267985 "DaMeSyFla".

\section{A Non-perturbative estimate of the $\operatorname{RCs} Z_{V}^{(\text {fact })}$ and $Z_{A}^{(\text {fact })}$}

\section{A.1 Axial Ward-Takahashi identity in the presence of electromagnetism}

For an isospin doublet $\psi \equiv\left(\psi_{1}, \psi_{2}\right)$ of mass-degenerate quarks the twisted-mass (TM) action including QED is given in the physical basis at maximal twist by [36, 38, 39]

$$
\mathcal{S}=a^{4} \sum_{x} \bar{\psi}(x)\left\{M(x, y)-\frac{1}{2 a} \sum_{\mu}\left[F_{\mu}(x, y)+B_{\mu}(x, y)\right]\right\} \psi(y),
$$


where

$$
\begin{aligned}
& M(x, y)=\left[m+i\left(4 \frac{r}{a}-m^{c r i t}\right) \gamma_{5} \tau_{3}\right] \delta(x, y), \\
& F_{\mu}(x, y)=\left(i r \gamma_{5} \tau_{3}-\gamma_{\mu}\right) U_{\mu}(x) E_{\mu}(x) \delta(x+a \hat{\mu}, y), \\
& B_{\mu}(x, y)=\left(i r \gamma_{5} \tau_{3}+\gamma_{\mu}\right) U_{\mu}^{\dagger}(y) E_{\mu}^{\dagger}(y) \delta(x-a \hat{\mu}, y),
\end{aligned}
$$

with $E_{\mu}(x)=e^{i e Q \mathcal{A}_{\mu}(x)}$ being the QED link, $\mathcal{A}_{\mu}(x)$ the photon field, $m$ the twisted bare quark mass (in QCD+QED), $m^{\text {crit }}$ the critical mass (in QCD+QED) and $Q \equiv \operatorname{diag}\left\{q_{1}, q_{2}\right\}$. Performing the local non-singlet axial rotation

$$
\begin{aligned}
& \psi(x) \rightarrow\left[1+i \alpha(x) \tau^{+} \gamma_{5}\right] \psi(x) \\
& \bar{\psi}(x) \rightarrow \bar{\psi}(x)\left[1+i \alpha(x) \tau^{+} \gamma_{5}\right]
\end{aligned}
$$

with $\tau^{+} \equiv \tau_{1}+i \tau_{2}$, one has

$$
\frac{\delta S}{\delta[i \alpha(x)]}=-\partial_{\mu} A_{\mu}(x)+2 m \bar{\psi}(x) \gamma_{5} \tau^{+} \psi(x)+X(x)=0,
$$

where $\partial_{\mu}$ is the backward derivative in the $\mu$ direction and

$$
\begin{aligned}
A_{\mu}(x)= & \frac{1}{2}\left[\bar{\psi}(x) \gamma_{\mu} \gamma_{5} \tau^{+} U_{\mu}(x) E_{\mu}(x) \psi(x+a \hat{\mu})+\text { h.c. }\right] \\
X(x)= & -\frac{1}{2 a} \sum_{\mu} \bar{\psi}(x-a \hat{\mu}) \gamma_{\mu} \gamma_{5} U_{\mu}(x-a \hat{\mu})\left[\tau^{+}, E_{\mu}(x-a \hat{\mu})\right] \psi(x)+\text { h.c. } \\
& -\frac{i r}{2 a} \sum_{\mu} \bar{\psi}(x) \tau^{+} \tau^{3} U_{\mu}(x) E_{\mu}(x) \psi(x+a \hat{\mu})+\text { h.c. } \\
& -\frac{i r}{2 a} \sum_{\mu} \bar{\psi}(x-a \hat{\mu}) \tau^{3} U_{\mu}(x-a \hat{\mu}) E_{\mu}(x-a \hat{\mu}) \tau^{+} \psi(x)+\text { h.c. }
\end{aligned}
$$

We now choose that the charges of the two quarks are the same, i.e. $q_{1}=q_{2}=q$. This implies that the isospin rotation $\tau^{+}$commutes with the QED link $E_{\mu}(x)$. Consequently, the first line in eq. (A.7) vanishes, while the second and third lines can be written as a backward derivative. Thus, eq. (A.5) becomes

$$
\partial_{\mu} \bar{A}_{\mu}^{\mathrm{TM}}(x)=2 m \bar{\psi}(x) \gamma_{5} \tau^{+} \psi(x),
$$

where $\bar{A}_{\mu}^{\mathrm{TM}}(x)$ is the 1-point split TM axial current

$$
\begin{aligned}
\bar{A}_{\mu}^{\mathrm{TM}}(x)= & \frac{1}{2}\left[\bar{\psi}(x) \gamma_{\mu} \gamma_{5} \tau^{+} U_{\mu}(x) E_{\mu}(x) \psi(x+a \hat{\mu})\right. \\
& \left.-i r \bar{\psi}(x) \tau^{+} \tau^{3} U_{\mu}(x) E_{\mu}(x) \psi(x+a \hat{\mu})+\text { h.c. }\right],
\end{aligned}
$$

which is conserved in the chiral limit and therefore it does not require any renormalization constant.

As is well known, the local current

$$
A_{\mu}^{\mathrm{TM}}(x) \equiv \bar{\psi}(x) \gamma_{\mu} \gamma_{5} \tau^{+} \psi(x)
$$


requires a multiplicative renormalization, given by the $\mathrm{RC} Z_{V}$ [38], in order to match the 1-point split TM axial current (A.9) in the continuum limit. Thus, provided the quark charges are the same, the local version of the TM axial Ward-Takahashi identity holds as well also in the presence of electromagnetism, viz.

$$
Z_{V} \partial_{\mu} A_{\mu}^{\mathrm{TM}}(x)=2 m P_{5}^{\mathrm{TM}}(x)+\mathcal{O}\left(a^{2}\right),
$$

where $P_{5}^{\mathrm{TM}}(x)$ is the bare pseudoscalar density operator $P_{5}^{\mathrm{TM}}(x) \equiv \bar{\psi}(x) \gamma_{5} \tau^{+} \psi(x)$.

\section{A.2 Determination of the RC $Z_{V}$}

Let's consider a pseudoscalar (PS) meson composed by the two mass- and charge-degenerate TM quarks $\left(\psi_{1}, \psi_{2}\right)$. Introducing the 2-point correlators

$$
\begin{aligned}
& C_{P_{5}^{\mathrm{TM}} P_{5}^{\mathrm{TM}}}(t)=\sum_{\vec{x}}\left\langle P_{5}^{\mathrm{TM}}(\vec{x}, t) P_{5}^{T M \dagger}(0)\right\rangle, \\
& C_{A_{0}^{\mathrm{TM}} P_{5}^{\mathrm{TM}}(t)}=\sum_{\vec{x}}\left\langle A_{0}^{\mathrm{TM}}(\vec{x}, t) P_{5}^{T M \dagger}(0)\right\rangle,
\end{aligned}
$$

eq. (A.11) implies

$$
Z_{V} \partial_{t} C_{A_{0}^{\mathrm{TM}} P_{5}^{\mathrm{TM}}}(t)=2 m C_{A_{0}^{\mathrm{TM}} P_{5}^{\mathrm{TM}}}(t)+\mathcal{O}\left(a^{2}\right) .
$$

At large Euclidean time distances $t \gg a$ one has

$$
\begin{aligned}
& C_{P_{5}^{\mathrm{TM}}} P_{5}^{\mathrm{TM}}(t) \underset{t \gg a}{\longrightarrow} \frac{\left|\left\langle 0\left|P_{5}^{\mathrm{TM}}\right| P S\right\rangle\right|^{2}}{2 M_{\mathrm{PS}}^{\mathrm{TM}}}\left[e^{-M_{\mathrm{PS}}^{\mathrm{TM}} t}+e^{-M_{\mathrm{PS}}^{\mathrm{TM}}(T-t)}\right], \\
& C_{A_{0}^{\mathrm{TM}} P_{5}^{\mathrm{TM}}}(t) \underset{t \gg a}{\longrightarrow} \frac{\left\langle 0\left|A_{0}^{\mathrm{TM}}\right| P S\right\rangle\left\langle 0\left|P_{5}^{\mathrm{TM}}\right| P S\right\rangle^{*}}{2 M_{\mathrm{PS}}^{\mathrm{TM}}}\left[e^{-M_{\mathrm{PS}}^{\mathrm{TM}} t}-e^{-M_{\mathrm{PS}}^{\mathrm{TM}}(T-t)}\right],
\end{aligned}
$$

and therefore the renormalization constant $Z_{V}$ can be determined in terms of the matrix elements $\left\langle 0\left|P_{5}^{\mathrm{TM}}\right| P S\right\rangle$ and $\left\langle 0\left|A_{0}^{\mathrm{TM}}\right| P S\right\rangle$ as

$$
Z_{V}=\frac{2 m}{M_{\mathrm{PS}}^{\mathrm{TM}}} \frac{\left\langle 0\left|P_{5}^{\mathrm{TM}}\right| P S\right\rangle}{\left\langle 0\left|A_{0}^{\mathrm{TM}}\right| P S\right\rangle} .
$$

For a generic quantity $O$ we consider the following expansion in $\alpha_{\mathrm{em}}$

$$
O=O^{(0)}+\delta O+\mathcal{O}\left(\alpha_{\mathrm{em}}^{2}\right)
$$

where $O^{(0)}$ and $\delta O$ indicates the quantity in absence of QED and at $\mathcal{O}\left(\alpha_{\mathrm{em}}\right)$, respectively. Thus, from eq. (A.17) one gets

$$
\frac{\delta Z_{V}}{Z_{V}^{(0)}}=\frac{\delta m}{m^{(0)}}+\frac{\delta\left\langle 0\left|P_{5}^{\mathrm{TM}}\right| P S\right\rangle}{\left\langle 0\left|P_{5}^{\mathrm{TM}}\right| P S^{(0)}\right\rangle}-\frac{\delta M_{\mathrm{PS}}^{\mathrm{TM}}}{M_{\mathrm{PS}^{(0)}}^{\mathrm{TM}}}-\frac{\delta\left\langle 0\left|A_{0}^{\mathrm{TM}}\right| P S\right\rangle}{\left\langle 0\left|A_{0}^{\mathrm{TM}}\right| P S^{(0)}\right\rangle},
$$

where $\delta m$ arises from the $\mathcal{O}\left(\alpha_{\mathrm{em}}\right)$ contribution to the bare quark mass given by eq. (5.6), viz.

$$
\delta m=m^{(0)} \frac{\alpha_{\mathrm{em}} q^{2}}{4 \pi}[6 \log (a \mu)-22.5954] .
$$


The quantities $\delta\left\langle 0\left|P_{5}^{\mathrm{TM}}\right| P S\right\rangle, \delta M_{\mathrm{PS}}^{\mathrm{TM}}$ and $\delta\left\langle 0\left|A_{0}^{\mathrm{TM}}\right| P S\right\rangle$ can be extracted from the contributions at $\mathcal{O}\left(\alpha_{\mathrm{em}}\right)$ to the 2 -point correlators (A.12)-(A.13). Putting $X=\left\{P_{5}^{\mathrm{TM}}, A_{0}^{\mathrm{TM}}\right\}$ one has

$$
\delta C_{X P_{5}^{\mathrm{TM}}}(t)=\delta C_{X P_{5}^{\mathrm{TM}}}^{\mathrm{exch}}(t)+\delta C_{X P_{5}^{\mathrm{TM}}}^{\mathrm{self}}(t)+\delta C_{X P_{5}^{\mathrm{TM}}}^{\mathrm{tad}}(t)+\delta C_{X P_{5}^{\mathrm{TM}}}^{\mathrm{PS}}(t)+\delta C_{X P_{5}}^{S}(t),
$$

where the superscripts refer to the exchange, self-energy, tadpole, PS and scalar insertions, introduced already in eqs. (5.1)-(5.5) and depicted in figure 8.

The ratio of eq. (A.21) with the lowest-order correlator $C_{X P_{5}^{\mathrm{TM}}}^{(0)}(t)$ behaves at large Euclidean time distances (up to around-the-world effect) as

$$
\frac{\delta C_{X P_{5}^{\mathrm{TM}}}(t)}{C_{X P_{5}^{\mathrm{TM}}}^{(0)}(t)} \underset{t \gg a}{\longrightarrow} \frac{\delta\left[\left\langle 0\left|P_{5}^{\mathrm{TM}}\right| P S\right\rangle\langle 0|X| P S\rangle\right]}{\left\langle 0\left|P_{5}^{\mathrm{TM}}\right| P S^{(0)}\right\rangle\left\langle 0|X| P S^{(0)}\right\rangle}-\delta M_{\mathrm{PS}}^{\mathrm{TM}} \cdot t
$$

from which all the ingredients entering eq. (A.19) can be calculated.

\section{A.3 Determination of the RCs $Z_{A}$ and $Z_{P} / Z_{S}$}

We now consider an isospin doublet $\psi^{\mathrm{OS}}=\left(\psi_{1}^{\mathrm{OS}}, \psi_{2}^{\mathrm{OS}}\right)$ of mass- and charge-degenerate quark fields regularized using the Osterwalder-Seiler (OS) prescription [40], i.e. the same value of the Wilson $r$-parameter is assumed for the two quarks. At maximal twist the local axial current

$$
A_{\mu}^{\mathrm{OS}}(x) \equiv \bar{\psi}^{\mathrm{OS}}(x) \gamma_{\mu} \gamma_{5} \tau^{+} \psi^{\mathrm{OS}}(x)
$$

requires a multiplicative $\mathrm{RC}$ given by $Z_{A}$ [38]. Once renormalized the matrix elements of the TM (A.10) and OS (A.23) axial currents can differ only by discretization effects and therefore one has

$$
Z_{A}\left\langle 0\left|A_{0}^{\mathrm{OS}}\right| P S\right\rangle=Z_{V}\left\langle 0\left|A_{0}^{\mathrm{TM}}\right| P S\right\rangle+\mathcal{O}\left(a^{2}\right)
$$

This implies

$$
\begin{aligned}
\frac{\delta Z_{A}}{Z_{A}^{(0)}} & =\frac{\delta Z_{V}}{Z_{V}^{(0)}}+\frac{\delta\left\langle 0\left|A_{0}^{\mathrm{TM}}\right| P S\right\rangle}{\left\langle 0\left|A_{0}^{\mathrm{TM}}\right| P S^{(0)}\right\rangle}-\frac{\delta\left\langle 0\left|A_{0}^{\mathrm{OS}}\right| P S\right\rangle}{\left\langle 0\left|A_{0}^{\mathrm{OS}}\right| P S^{(0)}\right\rangle} \\
& =\frac{\delta m}{m^{(0)}}+\frac{\delta\left\langle 0\left|P_{5}^{\mathrm{TM}}\right| P S\right\rangle}{\left\langle 0\left|P_{5}^{\mathrm{TM}}\right| P S^{(0)}\right\rangle}-\frac{\delta M_{\mathrm{PS}}^{\mathrm{TM}}}{M_{P S^{(0)}}^{\mathrm{TM}}}-\frac{\delta\left\langle 0\left|A_{0}^{\mathrm{OS}}\right| P S\right\rangle}{\left\langle 0\left|A_{0}^{\mathrm{OS}}\right| P S^{(0)}\right\rangle}
\end{aligned}
$$

where $\delta\left\langle 0\left|A_{0}^{\mathrm{OS}}\right| P S\right\rangle /\left\langle 0\left|A_{0}^{\mathrm{OS}}\right| P S^{(0)}\right\rangle$ can be determined from the relevant axial correlators computed in the OS regularization.

Similarly, the ratio $Z_{P} / Z_{S}$ of the RCs of the pseudoscalar and scalar densities can be determined by using the relation

$$
Z_{S}\left\langle 0\left|P_{5}^{\mathrm{OS}}\right| P S\right\rangle=Z_{P}\left\langle 0\left|P_{5}^{\mathrm{TM}}\right| P S\right\rangle+\mathcal{O}\left(a^{2}\right)
$$

As for the e.m. corrections $\delta Z_{P}$ and $\delta Z_{S}$ one has

$$
\frac{\delta Z_{P}}{Z_{P}^{(0)}}-\frac{\delta Z_{S}}{Z_{S}^{(0)}}=\frac{\delta\left\langle 0\left|P_{5}^{\mathrm{OS}}\right| P S\right\rangle}{\left\langle 0\left|P_{5}^{\mathrm{OS}}\right| P S^{(0)}\right\rangle}-\frac{\delta\left\langle 0\left|P_{5}^{\mathrm{TM}}\right| P S\right\rangle}{\left\langle 0\left|P_{5}^{\mathrm{TM}}\right| P S^{(0)}\right\rangle},
$$

which, however, does not allow to determine separately the two corrections $\delta Z_{P}$ and $\delta Z_{S}$. For this reason we will not investigate eq. (A.27) numerically. 


\begin{tabular}{|c|c|c|}
\hline$\beta$ & $Z_{V}^{\text {(fact) }}$ & $Z_{A}^{\text {(fact) }}$ \\
\hline 1.90 & $1.027(5)$ & $0.85(5)$ \\
\hline 1.95 & $1.033(4)$ & $0.93(5)$ \\
\hline 2.10 & $1.034(3)$ & $0.87(6)$ \\
\hline
\end{tabular}

Table 5. Results for $Z_{V}^{(\text {fact) }}$ and $Z_{A}^{\text {(fact) }}$ (see eq. (A.28)) obtained at the three values of the inverse bare lattice coupling $\beta$ corresponding to the gauge ensembles of table 1.

\section{A.4 Numerical results}

In order to get a first non-perturbative estimate of the RCS $Z_{V}$ and $Z_{A}$ in QCD+QED we have calculated the r.h.s. of eqs. (A.19) and (A.25) using for the (bare) quark mass $m$ the values of the strange quark masses reported in table 1 for the three values of the inverse lattice coupling $\beta$ of the ETMC ensembles. As in section 5, we introduce the correction factors $Z_{V}^{(\mathrm{fact})}$ and $Z_{A}^{(\mathrm{fact})}$ to the "naive factorization" approximation by defining

$$
\delta Z_{V}=Z_{V}^{(0)} \cdot Z_{V}^{(\mathrm{em})} \cdot Z_{V}^{(\mathrm{fact})}, \quad \delta Z_{A}=Z_{A}^{(0)} \cdot Z_{A}^{(\mathrm{em})} \cdot Z_{A}^{(\mathrm{fact})},
$$

where $Z_{V(A)}^{(\mathrm{em})}$ is the one-loop perturbative estimate of the QED effect at order $\mathcal{O}\left(\alpha_{s}^{0}\right)$ in the strong coupling. Using for the latter ones the perturbative findings $Z_{V}^{(\mathrm{em})}=-20.6178 \alpha_{\mathrm{em}} q^{2} /(4 \pi)$ and $Z_{A}^{(\mathrm{em})}=-15.7963 \alpha_{\mathrm{em}} q^{2} /(4 \pi)$ from refs. [46, 47], our results for $Z_{V}^{(\mathrm{fact})}$ and $Z_{A}^{\text {(fact) }}$ are collected in table 5 . It can be seen that the dependence on the lattice spacing is quite mild within the uncertainties. The averages of the results of table 5 (according to eq. (28) of ref. [34]) are: $Z_{V}^{(\text {fact) }}=1.031 \pm 0.005$ and $Z_{A}^{(\text {fact })}=0.88 \pm 0.06$. Given the exploratory nature of the present non-perturbative determination of QCD+QED renormalization constants, we prefer to quote as our estimates for $Z_{V}^{(\mathrm{fact})}$ and $Z_{A}^{(\mathrm{fact})}$ the values

$$
Z_{V}^{(\mathrm{fact})}=1.03 \pm 0.01, \quad Z_{A}^{(\mathrm{fact})}=0.9 \pm 0.1
$$

which cover the spread of the results given in table 5 .

Open Access. This article is distributed under the terms of the Creative Commons Attribution License (CC-BY 4.0), which permits any use, distribution and reproduction in any medium, provided the original author(s) and source are credited.

\section{References}

[1] Muon G-2 collaboration, G.W. Bennett et al., Final Report of the Muon E821 Anomalous Magnetic Moment Measurement at BNL, Phys. Rev. D 73 (2006) 072003 [hep-ex/0602035] [INSPIRE].

[2] Particle Data Group collaboration, C. Patrignani et al., Review of Particle Physics, Chin. Phys. C 40 (2016) 100001 [INSPIRE].

[3] Muon $g-2$ collaboration, I. Logashenko et al., The Measurement of the Anomalous Magnetic Moment of the Muon at Fermilab, J. Phys. Chem. Ref. Data 44 (2015) 031211 [INSPIRE]. 
[4] E34 collaboration, M. Otani, Design of the J-PARC MUSE H-line for the Muon g-2/EDM Experiment at J-PARC (E34), JPS Conf. Proc. 8 (2015) 025010 [InSPIRE].

[5] F. Jegerlehner and A. Nyffeler, The Muon g-2, Phys. Rept. 477 (2009) 1 [arXiv: 0902.3360] [INSPIRE].

[6] M. Davier, A. Hoecker, B. Malaescu and Z. Zhang, Reevaluation of the Hadronic Contributions to the Muon $g-2$ and to $\alpha\left(M_{Z}^{2}\right)$, Eur. Phys. J. C 71 (2011) 1515 [Erratum ibid. C 72 (2012) 1874] [arXiv:1010.4180] [INSPIRE].

[7] K. Hagiwara, R. Liao, A.D. Martin, D. Nomura and T. Teubner, $(g-2)_{\mu}$ and $\alpha\left(M_{Z}^{2}\right)$ re-evaluated using new precise data, J. Phys. G 38 (2011) 085003 [arXiv:1105.3149] [INSPIRE].

[8] B.e. Lautrup, A. Peterman and E. de Rafael, Recent developments in the comparison between theory and experiments in quantum electrodynamics, Phys. Rept. 3 (1972) 193 [INSPIRE].

[9] E. de Rafael, Hadronic contributions to the muon g-2 and low-energy QCD, Phys. Lett. B 322 (1994) 239 [hep-ph/9311316] [INSPIRE].

[10] T. Blum, Lattice calculation of the lowest order hadronic contribution to the muon anomalous magnetic moment, Phys. Rev. Lett. 91 (2003) 052001 [hep-lat/0212018] [INSPIRE].

[11] P. Boyle, L. Del Debbio, E. Kerrane and J. Zanotti, Lattice Determination of the Hadronic Contribution to the Muon $g-2$ using Dynamical Domain Wall Fermions, Phys. Rev. D 85 (2012) 074504 [arXiv:1107.1497] [INSPIRE].

[12] M. Della Morte, B. Jager, A. Juttner and H. Wittig, Towards a precise lattice determination of the leading hadronic contribution to $(g-2)_{\mu}$, JHEP 03 (2012) 055 [arXiv:1112.2894] [INSPIRE].

[13] ETM collaboration, F. Burger, X. Feng, G. Hotzel, K. Jansen, M. Petschlies and D.B. Renner, Four-Flavour Leading-Order Hadronic Contribution To The Muon Anomalous Magnetic Moment, JHEP 02 (2014) 099 [arXiv: 1308.4327] [INSPIRE].

[14] HPQCD collaboration, B. Chakraborty et al., Strange and charm quark contributions to the anomalous magnetic moment of the muon, Phys. Rev. D 89 (2014) 114501 [arXiv: 1403.1778] [INSPIRE].

[15] B. Chakraborty, C. Davies, P.G. de Oliveira, J. Koponen and G.P. Lepage, Lattice calculation of the HVP contribution to the anomalous magnetic moment of muon, PoS(LATTICE 2015) 108 [arXiv: 1511.05870] [INSPIRE].

[16] G. Bali and G. Endrödi, Hadronic vacuum polarization and muon $g-2$ from magnetic susceptibilities on the lattice, Phys. Rev. D 92 (2015) 054506 [arXiv:1506.08638] [InSPIRE].

[17] B. Chakraborty, C.T.H. Davies, J. Koponen, G.P. Lepage, M.J. Peardon and S.M. Ryan, Estimate of the hadronic vacuum polarization disconnected contribution to the anomalous magnetic moment of the muon from lattice QCD, Phys. Rev. D 93 (2016) 074509 [arXiv: 1512.03270] [INSPIRE].

[18] T. Blum et al., Calculation of the hadronic vacuum polarization disconnected contribution to the muon anomalous magnetic moment, Phys. Rev. Lett. 116 (2016) 232002 [arXiv: 1512.09054] [INSPIRE].

[19] RBC/UKQCD collaboration, T. Blum et al., Lattice calculation of the leading strange quark-connected contribution to the muon g-2, JHEP 04 (2016) 063 [Erratum ibid. 05 (2017) 034] [arXiv:1602.01767] [INSPIRE]. 
[20] B. Chakraborty, C.T.H. Davies, P.G. de Oliviera, J. Koponen, G.P. Lepage and R.S. Van de Water, The hadronic vacuum polarization contribution to $a_{\mu}$ from full lattice $Q C D$, Phys. Rev. D 96 (2017) 034516 [arXiv:1601.03071] [INSPIRE].

[21] M. Della Morte et al., The hadronic vacuum polarization contribution to the muon $g-2$ from lattice QCD, JHEP 10 (2017) 020 [arXiv:1705.01775] [INSPIRE].

[22] J. Green, O. Gryniuk, G. von Hippel, H.B. Meyer and V. Pascalutsa, Lattice QCD calculation of hadronic light-by-light scattering, Phys. Rev. Lett. 115 (2015) 222003 [arXiv: 1507.01577] [INSPIRE].

[23] T. Blum, N. Christ, M. Hayakawa, T. Izubuchi, L. Jin and C. Lehner, Lattice Calculation of Hadronic Light-by-Light Contribution to the Muon Anomalous Magnetic Moment, Phys. Rev. D 93 (2016) 014503 [arXiv: 1510.07100] [inSPIRE].

[24] G. Colangelo, M. Hoferichter, M. Procura and P. Stoffer, Dispersion relation for hadronic light-by-light scattering: theoretical foundations, JHEP 09 (2015) 074 [arXiv:1506.01386] [INSPIRE].

[25] J. Bijnens and J. Relefors, Pion light-by-light contributions to the muon g- 2, JHEP 09 (2016) 113 [arXiv:1608.01454] [INSPIRE].

[26] G. Colangelo, M. Hoferichter, M. Procura and P. Stoffer, Dispersion relation for hadronic light-by-light scattering: two-pion contributions, JHEP 04 (2017) 161 [arXiv:1702.07347] [INSPIRE].

[27] G.M. de Divitiis et al., Isospin breaking effects due to the up-down mass difference in Lattice QCD, JHEP 04 (2012) 124 [arXiv:1110.6294] [INSPIRE].

[28] RM123 collaboration, G.M. de Divitiis et al., Leading isospin breaking effects on the lattice, Phys. Rev. D 87 (2013) 114505 [arXiv: 1303.4896] [InSPIRE].

[29] D. Giusti et al., Leading isospin-breaking corrections to pion, kaon and charmed-meson masses with Twisted-Mass fermions, Phys. Rev. D 95 (2017) 114504 [arXiv:1704.06561] [INSPIRE].

[30] V. Lubicz et al., Electromagnetic corrections to the leptonic decay rates of charged pseudoscalar mesons: lattice results, PoS (LATTICE2016) 290 [arXiv:1610.09668] [INSPIRE].

[31] N. Tantalo, V. Lubicz, G. Martinelli, C.T. Sachrajda, F. Sanfilippo and S. Simula, Electromagnetic corrections to leptonic decay rates of charged pseudoscalar mesons: finite-volume effects, arXiv: 1612.00199 [INSPIRE].

[32] P. Boyle et al., Isospin breaking corrections to meson masses and the hadronic vacuum polarization: a comparative study, JHEP 09 (2017) 153 [arXiv:1706.05293] [INSPIRE].

[33] D. Bernecker and H.B. Meyer, Vector Correlators in Lattice QCD: Methods and applications, Eur. Phys. J. A 47 (2011) 148 [arXiv:1107.4388] [inSPIRE].

[34] European Twisted Mass collaboration, N. Carrasco et al., Up, down, strange and charm quark masses with $N_{f}=2+1+1$ twisted mass lattice QCD, Nucl. Phys. B 887 (2014) 19 [arXiv: 1403.4504] [INSPIRE].

[35] Y. Iwasaki, Renormalization Group Analysis of Lattice Theories and Improved Lattice Action: Two-Dimensional Nonlinear $O(N) \sigma$-model, Nucl. Phys. B 258 (1985) 141 [InSPIRE].

[36] Alpha collaboration, R. Frezzotti, P.A. Grassi, S. Sint and P. Weisz, Lattice QCD with a chirally twisted mass term, JHEP 08 (2001) 058 [hep-lat/0101001] [INSPIRE]. 
[37] R. Frezzotti and G.C. Rossi, Twisted mass lattice QCD with mass nondegenerate quarks, Nucl. Phys. Proc. Suppl. 128 (2004) 193 [hep-lat/0311008] [INSPIRE].

[38] R. Frezzotti and G.C. Rossi, Chirally improving Wilson fermions. 1. O(a) improvement, JHEP 08 (2004) 007 [hep-lat/0306014] [INSPIRE].

[39] R. Frezzotti and G.C. Rossi, Chirally improving Wilson fermions. 2. Four-quark operators, JHEP 10 (2004) 070 [hep-lat/0407002] [INSPIRE].

[40] K. Osterwalder and E. Seiler, Gauge Field Theories on the Lattice, Annals Phys. 110 (1978) 440 [INSPIRE].

[41] F. Burger, G. Hotzel, K. Jansen and M. Petschlies, The hadronic vacuum polarization and automatic $\mathcal{O}($ a) improvement for twisted mass fermions, JHEP 03 (2015) 073 [arXiv: 1412.0546] [INSPIRE].

[42] K.G. Chetyrkin, J.H. Kuhn and M. Steinhauser, Three loop polarization function and $O\left(\alpha_{S}^{2}\right)$ corrections to the production of heavy quarks, Nucl. Phys. B 482 (1996) 213 [hep-ph/9606230] [INSPIRE].

[43] S. Simula et al., QED corrections to meson decay rates in $L Q C D$, PRACE project Pra-102693 [http://www.prace-ri.eu/prace-10th-project-call/\#Fundamental].

[44] UKQCD collaboration, C. McNeile and C. Michael, Decay width of light quark hybrid meson from the lattice, Phys. Rev. D 73 (2006) 074506 [hep-lat/0603007] [INSPIRE].

[45] C. Alexandrou, S. Bacchio, J. Finkenrath, A. Frommer, K. Kahl and M. Rottmann, Adaptive Aggregation-based Domain Decomposition Multigrid for Twisted Mass Fermions, Phys. Rev. D 94 (2016) 114509 [arXiv: 1610.02370] [INSPIRE].

[46] G. Martinelli and Y.-C. Zhang, The Connection Between Local Operators on the Lattice and in the Continuum and Its Relation to Meson Decay Constants, Phys. Lett. B 123 (1983) 433 [INSPIRE].

[47] S. Aoki, K.-i. Nagai, Y. Taniguchi and A. Ukawa, Perturbative renormalization factors of bilinear quark operators for improved gluon and quark actions in lattice QCD, Phys. Rev. D 58 (1998) 074505 [hep-lat/9802034] [INSPIRE].

[48] M. Hayakawa and S. Uno, QED in finite volume and finite size scaling effect on electromagnetic properties of hadrons, Prog. Theor. Phys. 120 (2008) 413 [arXiv:0804.2044] [INSPIRE].

[49] V. Lubicz, G. Martinelli, C.T. Sachrajda, F. Sanfilippo, S. Simula and N. Tantalo, Finite-Volume QED Corrections to Decay Amplitudes in Lattice QCD, Phys. Rev. D 95 (2017) 034504 [arXiv: 1611.08497] [INSPIRE].

[50] Z. Davoudi and M.J. Savage, Finite-Volume Electromagnetic Corrections to the Masses of Mesons, Baryons and Nuclei, Phys. Rev. D 90 (2014) 054503 [arXiv:1402.6741] [INSPIRE]. 